Budker INP 2002-38

DESY 02-074

DFCAL-TH $02 / 2$

June 2002

\title{
A PROOF OF FULFILLMENT OF THE STRONG BOOTSTRAP CONDITION *
}

\author{
V.S. Fadin ${ }^{a \dagger}$ and A. Papa ${ }^{b}$ \\ ${ }^{a}$ Budker Institute for Nuclear Physics, 630090 Novosibirsk, Russia \\ and Novosibirsk State University, 630090 Novosibirsk, Russia \\ ${ }^{b}$ Dipartimento di Fisica, Università della Calabria, \\ and Istituto Nazionale di Fisica Nucleare, Gruppo collegato di Cosenza, \\ I-87036 Arcavacata di Rende, Cosenza, Italy
}

\begin{abstract}
It is shown that the kernel of the BFKL equation for the octet color state of two Reggeized gluons satisfies the strong bootstrap condition in the next-to-leading order. This condition is much more restrictive than the one obtained from the requirement of Reggeized form for the elastic scattering amplitudes in the next-to-leading approximation. It is necessary, however, for self-consistency of the assumption of Reggeized form of the production amplitudes in multi-Regge kinematics, which are used in the derivation of the BFKL equation. The fulfillment of the strong bootstrap condition for the kernel opens a way to a rigorous proof of the BFKL equation in the next-to-leading approximation.
\end{abstract}

* Work supported in part by the Ministero dell'Istruzione, dell'Università e della Ricerca, in part by INTAS and in part by the Russian Fund of Basic Researches.

${ }^{\dagger}$ e-mail address: FADIN@INP.NSK.SU

$\ddagger$ e-mail address: PAPA@FIS.UNICAL.IT 


\section{Introduction}

The BFKL approach [1] to the description of processes at large c.m.s. energy $\sqrt{s}$ and fixed momentum transfer $\sqrt{-} t$ is based on the gluon Reggeization. In this approach the scattering amplitudes are given by the convolution of the impact factors of the scattered particles and the Green function for the Reggeon-Reggeon scattering. The Pomeron, which determines the high energy behavior of cross sections, and the Odderon, responsible for the difference of particle and antiparticle cross sections, appear in this approach as compound states of two and three Reggeized gluons respectively.

Originally the BFKL approach was developed in the leading logarithmic approximation (LLA), that means resummation of terms $\left(\alpha_{s} \ln s\right)^{n}$. Now for the forward scattering $(t=0$ and color singlet in the $t$-channel) the kernel of the equation for the two-Reggeon Green function is known [2, 3] in the next-to-leading order (NLO). The calculation of the NLO kernel for the non-forward scattering [4] is not far from completion (see [5, 6]). The impact factors of gluons [7] and quarks [8] are calculated in the NLO and the impact factors of the physical (color singlet) particles are under investigation [9, 10, 11, 12, 13].

Note that although initially the gluon Reggeization appeared as the assumption used in the derivation of the BFKL equation [1], later it was proved in the LLA [14]. The key to the proof is given by the properties of the amplitudes with gluon quantum numbers (color octet with negative signature) in the $t$-channels.

Whereas two Reggeized gluons in the color singlet state create the BFKL Pomeron, in the antisymmetric color octet state they must reproduce the Reggeon itself. This is the requirement of self-consistency of the BFKL approach, since it is based on the gluon Reggeization. The bootstrap conditions for the color octet impact factors and the BFKL kernel in the NLO, arising from the application of this requirement to the elastic scattering amplitudes, were obtained in [4. The condition for the impact factors was checked in [7] and [8] in the cases of gluon and quark, respectively, and was proved to be satisfied at arbitrary space-time dimension $D$ both for the helicity conserving and non-conserving parts of the impact factors, as well as the bootstrap condition for the quark part of the kernel, which was analyzed in [5]. The fulfillment of the bootstrap condition for the gluon part of the kernel was proved in the limit $D \rightarrow 4$ [15]. The evident reason for this restriction was that the gluon part of the color octet kernel was known at that time [6] only in this limit. Note that now the kernel at arbitrary $D$ is calculated [16] and it is possible to check the bootstrap condition at arbitrary $D$.

Although the bootstrap conditions arising from the elastic scattering amplitudes are rather restrictive, their fulfillment can not justify completely the BFKL equation. Evidently, the bootstrap conditions must be satisfied for all amplitudes involved into the derivation of the BFKL equation, since they were supposed to have (multi) Regge form, i.e. for the amplitudes of production of any number of particles in the multi-Regge and (in the NLA) quasi multi-Regge kinematics. The fulfillment of all these conditions gives the basis on which the proof of Reggeization was constructed in the LLA [14. An analogous proof can be constructed in the next-to-leading approximation (NLA) as well [17]. 
The requirement of bootstrap for the multi-particle production leads, in particular, [17] to stronger restrictions on octet impact factors and kernel, than it follows from the elastic amplitudes. These restrictions are just the so called strong bootstrap conditions, which were suggested, without derivation, in [18, 19]. They can be presented in the form [20]:

$$
\begin{gathered}
\Phi_{A^{\prime} A}^{a}\left(\overrightarrow{q_{1}}, \vec{q}\right)=\frac{-i g \sqrt{N}}{2} \Gamma_{A^{\prime} A}^{a}(q) R\left(\vec{q}_{1}, \vec{q}\right), \\
\int \frac{d^{D-2} q_{2}}{{\overrightarrow{q_{2}}}_{2}^{2} \vec{q}_{2}^{\prime 2}} \mathcal{K}\left(\vec{q}_{1}, \overrightarrow{q_{2}} ; \vec{q}\right) R\left(\vec{q}_{2}, \vec{q}\right)=\omega(t) R\left(\vec{q}_{1}, \vec{q}\right) .
\end{gathered}
$$

Here and in the following $N_{c}$ is the number of colors, $\vec{q}$ is the transverse part of the total transferred momentum, $t=-\vec{q}^{2}, \vec{q}_{i}$ and $\vec{q}_{i}^{\prime} \equiv \vec{q}-\vec{q}_{i}, i=1,2$, are the momenta of the Reggeized gluons; $\Phi_{A^{\prime} A}^{a}$ is the octet impact factor for the $A \rightarrow A^{\prime}$ transition, $\Gamma_{A^{\prime} A}^{a}$ is the Reggeon vertex, $R\left(\vec{q}_{1}, \vec{q}\right)$ appears as an universal (i.e. independent on properties of the scattered particles) function, which occurs to be the eigenfunction of the octet kernel $\mathcal{K}\left(\vec{q}_{1}, \vec{q}_{2} ; \vec{q}\right)$ with eigenvalue $\omega(t)=j(t)-1, j(t)$ is the gluon Regge trajectory. Note that here we have changed the normalization of $R$ compared to [20, so that now in the leading order $R=R^{(0)}=1$. Fulfillment of (1.1) in this order is immediately seen from explicit expressions for the impact factors and vertices, which are constant in this order. The remarkable property of the color octet impact factors and Reggeon vertices exhibited by this relation is that their ratio is a process-independent function. This property becomes quite nontrivial in the NLO. It was verified by comparison of this ratio for quarks and gluons in [20], where the NLO contribution $R^{(1)}$ to the function $R$ was determined.

Taking into account a general relation [4]:

$$
\mathcal{K}\left(\vec{q}_{1}, \vec{q}_{2} ; \vec{q}\right)=\left(\omega\left(-\vec{q}_{1}^{2}\right)+\omega\left(-\vec{q}_{1}^{\prime 2}\right)\right) \vec{q}_{1}^{2} \vec{q}_{1}^{\prime 2} \delta^{(D-2)}\left(\vec{q}_{1}-\vec{q}_{2}\right)+\mathcal{K}_{r}\left(\vec{q}_{1}, \vec{q}_{2} ; \vec{q}\right),
$$

where $\mathcal{K}_{r}$ is the part of the kernel related to the real particle production, the condition (1.2) can be rewritten as

$$
\int \frac{d^{D-2} q_{2}}{\vec{q}_{2}^{2} \vec{q}_{2}^{\prime 2}} \mathcal{K}_{r}\left(\vec{q}_{1}, \vec{q}_{2} ; \vec{q}\right) R\left(\vec{q}_{2}, \vec{q}\right)=\left(\omega(t)-\omega\left(t_{1}\right)-\omega\left(t_{1}^{\prime}\right)\right) R\left(\vec{q}_{1}, \vec{q}\right)
$$

where $t_{i}=q_{i}^{2}=-\vec{q}_{i}^{2}, i=1,2$. The fulfillment of this relation in the leading order (remember that $\left.R^{(0)}=1\right)$ :

$$
\int \frac{d^{D-2} q_{2}}{\vec{q}_{2}^{2} \vec{q}_{2}^{\prime 2}} \mathcal{K}_{r}^{(0)}\left(\vec{q}_{1}, \vec{q}_{2} ; \vec{q}\right)=\omega^{(1)}(t)-\omega^{(1)}\left(t_{1}\right)-\omega^{(1)}\left(t_{1}^{\prime}\right)
$$

is evident, taking into account the leading order expressions for the "real" part of the kernel

$$
\mathcal{K}_{r}^{(0)}\left(\vec{q}_{1}, \overrightarrow{q_{2}} ; \vec{q}\right)=\frac{g^{2} N_{c}}{2(2 \pi)^{D-1}} f_{B}\left(\vec{q}_{1}, \overrightarrow{q_{2}} ; \vec{q}\right), \quad f_{B}\left(\overrightarrow{q_{1}}, \overrightarrow{q_{2}} ; \vec{q}\right)=\frac{\vec{q}_{1}^{2}{\overrightarrow{q_{2}}}^{2}+\vec{q}_{2}^{2} \vec{q}_{1}^{\prime 2}}{\vec{k}^{2}}-\vec{q}^{2}
$$

where $\vec{k}=\vec{q}_{1}-\vec{q}_{2}$, and the trajectory

$$
\omega^{(1)}(t)=\frac{g^{2} N_{c} t}{2(2 \pi)^{D-1}} \int \frac{d^{D-2} q_{1}}{\vec{q}_{1}^{2} \vec{q}_{1}^{\prime 2}}=-g^{2} \frac{N_{c} \Gamma(1-\epsilon)}{(4 \pi)^{D / 2}} \frac{\Gamma^{2}(\epsilon)}{\Gamma(2 \epsilon)}\left(\vec{q}^{2}\right)^{\epsilon},
$$


with $D=4+2 \epsilon$.

In this paper we prove the fulfillment of the bootstrap condition (1.4) in the NLO. It is explicitly formulated and simplified in the next Section; Section 3 contains the proof. The details of the calculations are given in the Appendices. The significance of the result is discussed in Section 4.

\section{Formulation and simplification of the bootstrap con- dition}

Let us work in pure gluodynamics, since the fulfillment of the strong bootstrap condition for the quark part of the kernel simply follows from the results of [5, 19, 21]. Then the condition (1.1), with account of $R^{(0)}=1$ and (1.5) gives us in the NLO

$$
\begin{gathered}
\int \frac{d^{D-2} q_{2}}{{\overrightarrow{q_{2}}}_{2}^{2} \vec{q}_{2}^{\prime 2}}\left[\mathcal{K}_{r}^{(1)}\left(\vec{q}_{1}, \overrightarrow{q_{2}}, \vec{q}\right)+\mathcal{K}_{r}^{(0)}\left(\vec{q}_{1}, \overrightarrow{q_{2}}, \vec{q}\right)\left(R^{(1)}\left(\overrightarrow{q_{2}}, \vec{q}\right)-R^{(1)}\left(\overrightarrow{q_{1}}, \vec{q}\right)\right)\right. \\
=\omega^{(2)}(t)-\omega^{(2)}\left(t_{1}\right)-\omega^{(2)}\left(t_{1}^{\prime}\right) .
\end{gathered}
$$

Here $\mathcal{K}_{r}^{(1)}, \omega^{(2)}$ and $R^{(1)}$ are the NLO contributions to the "real" part of the kernel $\mathcal{K}$ of the BFKL equation for the octet color representation in the $t$-channel, to the gluon Regge

trajectory and to the function $R$ determined by the bootstrap condition for the impact factors (1.1). We have

$$
\omega^{(2)}(t)=\left[\frac{g^{2} N_{c} \Gamma(1-\epsilon)\left(\vec{q}^{2}\right)^{\epsilon}}{(4 \pi)^{D / 2} \epsilon}\right]^{2}\left[\frac{11}{3}+\left(2 \psi^{\prime}(1)-\frac{67}{9}\right) \epsilon+\left(\frac{404}{27}+\psi^{\prime \prime}(1)-\frac{22}{3} \psi^{\prime}(1)\right) \epsilon^{2}\right]
$$

where $\psi(z)=\Gamma^{\prime}(z) / \Gamma(z)$ and all non-vanishing terms for $\epsilon \rightarrow 0$ are held. Although the expression of $\omega^{(2)}(t)$ in terms of integrals in the transverse momentum space was obtained for arbitrary $\epsilon$ [22], an explicit expression for it is known only at $\epsilon \rightarrow 0$ [23, 24, 25].

The NLO correction to $R$ is [20]

$$
\begin{aligned}
R^{(1)}\left(\vec{q}_{1}, \vec{q}\right)= & \frac{\omega^{(1)}(t)}{2}\left[\frac{\epsilon \Gamma(1+2 \epsilon)\left(\vec{q}^{2}\right)^{1-\epsilon}}{2 \Gamma^{2}(1+\epsilon)} \int \frac{d^{D-2} k}{\Gamma(1-\epsilon) \pi^{1+\epsilon}} \frac{\ln \left(\vec{q}^{2} / \vec{k}^{2}\right)}{\left(\vec{k}-\vec{q}_{1}\right)^{2}\left(\vec{k}+\vec{q}_{1}^{\prime}\right)^{2}}\right. \\
+\left(\left(\frac{\vec{q}_{1}^{2}}{\vec{q}^{2}}\right)^{\epsilon}+\right. & \left.\left(\frac{\vec{q}_{1}^{\prime 2}}{\vec{q}^{2}}\right)^{\epsilon}-1\right)\left(\frac{1}{2 \epsilon}+\psi(1+2 \epsilon)-\psi(1+\epsilon)+\frac{11+7 \epsilon}{2(1+2 \epsilon)(3+2 \epsilon)}\right) \\
& \left.-\frac{1}{2 \epsilon}+\psi(1)+\psi(1+\epsilon)-\psi(1-\epsilon)-\psi(1+2 \epsilon)\right] .
\end{aligned}
$$

The NLO corrections to the part of the kernel related with the real particle production, $\mathcal{K}_{r}$, can be presented [15] as follows in the limit $\epsilon \rightarrow 0$ :

$$
\mathcal{K}_{r}^{(1)}=\frac{\bar{g}^{4}}{\pi^{1+\epsilon} \Gamma(1-\epsilon)}\left(\mathcal{K}_{1}+\mathcal{K}_{2}+\mathcal{K}_{3}\right), \quad \bar{g}^{2} \equiv \frac{g^{2} N_{c} \Gamma(1-\epsilon)}{(4 \pi)^{D / 2}},
$$


with

$$
\mathcal{K}_{1}=-f_{B}\left(\overrightarrow{q_{1}}, \overrightarrow{q_{2}} ; \vec{q}\right) \frac{\left(\vec{k}^{2}\right)^{\epsilon}}{\epsilon}\left[\frac{11}{3}+\left(2 \psi^{\prime}(1)-\frac{67}{9}\right) \epsilon+\left(\frac{404}{27}+7 \psi^{\prime \prime}(1)-\frac{11}{3} \psi^{\prime}(1)\right) \epsilon^{2}\right],
$$

where $\vec{k}=\vec{q}_{1}-\vec{q}_{2}$,

$$
\begin{gathered}
\mathcal{K}_{2}=\left\{\vec{q}^{2}\left[\frac{11}{6} \ln \left(\frac{\vec{q}_{1}^{2} \vec{q}_{2}^{2}}{\vec{q}^{2} \vec{k}^{2}}\right)+\frac{1}{4} \ln \left(\frac{\vec{q}_{1}^{2}}{\vec{q}^{2}}\right) \ln \left(\frac{\vec{q}_{1}^{2}}{\vec{q}^{2}}\right)+\frac{1}{4} \ln \left(\frac{\vec{q}_{2}^{2}}{\vec{q}^{2}}\right) \ln \left(\frac{\vec{q}_{2}^{\prime 2}}{\vec{q}^{2}}\right)+\frac{1}{4} \ln ^{2}\left(\frac{\vec{q}_{1}^{2}}{\vec{q}_{2}^{2}}\right)\right]\right. \\
\left.-\frac{\vec{q}_{1}^{2} \vec{q}_{2}^{\prime 2}+\vec{q}_{2}^{2} \vec{q}_{1}^{\prime 2}}{2 \vec{k}^{2}} \ln ^{2}\left(\frac{\vec{q}_{1}^{2}}{\vec{q}_{2}^{2}}\right)+\frac{\vec{q}_{1}^{2} \vec{q}_{2}^{\prime 2}-\vec{q}_{2}^{2} \vec{q}_{1}^{\prime 2}}{\vec{k}^{2}} \ln \left(\frac{\vec{q}_{1}^{2}}{\vec{q}_{2}^{2}}\right)\left(\frac{11}{6}-\frac{1}{4} \ln \left(\frac{\vec{q}_{1}^{2} \vec{q}_{2}^{2}}{\vec{k}^{4}}\right)\right)\right\}+\left\{\vec{q}_{i} \leftrightarrow \vec{q}_{i}^{\prime}\right\}, \\
\mathcal{K}_{3}=\left\{\frac{1}{2}\left[\vec{q}^{2}\left(\vec{k}^{2}-\vec{q}_{1}^{2}-\vec{q}_{2}^{2}\right)+2 \vec{q}_{1}^{2} \vec{q}_{2}^{2}-\vec{q}_{1}^{2} \vec{q}_{2}^{\prime 2}-\vec{q}_{2}^{2} \vec{q}_{1}^{\prime 2}+\frac{\vec{q}_{1}^{2} \vec{q}_{2}^{\prime 2}-\vec{q}_{2}^{2} \vec{q}_{1}^{\prime 2}}{\vec{k}^{2}}\left(\vec{q}_{1}^{2}-\vec{q}_{2}^{2}\right)\right]\right. \\
\left.\quad \times \int_{0}^{1} \frac{d x}{\left(\vec{q}_{1}(1-x)+\vec{q}_{2} x\right)^{2}} \ln \left(\frac{\vec{q}_{1}^{2}(1-x)+\vec{q}_{2}^{2} x}{\vec{k}^{2} x(1-x)}\right)\right\}+\left\{\vec{q}_{i} \leftrightarrow \vec{q}_{i}^{\prime}\right\} .
\end{gathered}
$$

Using the result of [7] for the integral in (2.10) in the limit $\epsilon \rightarrow 0$,

$$
\left(\vec{q}^{2}\right)^{1-\epsilon} \int \frac{d^{D-2} k}{\Gamma(1-\epsilon) \pi^{1+\epsilon}} \frac{\ln \left(\vec{q}^{2} / \vec{k}^{2}\right)}{\left(\vec{k}-\vec{q}_{1}\right)^{2}\left(\vec{k}+\vec{q}_{1}^{\prime}\right)^{2}}=-\frac{1}{\epsilon} \ln \left(\frac{\vec{q}_{1}^{2} \vec{q}_{1}^{\prime 2}}{\left(\vec{q}^{2}\right)^{2}}\right)-\frac{1}{2} \ln ^{2}\left(\frac{\vec{q}_{1}^{2}}{\vec{q}_{1}^{\prime 2}}\right)+O(\epsilon),
$$

Eqs. (2.9), (2.10), (2.11), and taking into account the properties of the kernel

$$
f_{B}\left(\vec{q}_{1}, \overrightarrow{0} ; \vec{q}\right)=f_{B}\left(\overrightarrow{q_{1}}, \vec{q} ; \vec{q}\right)=\mathcal{K}_{2}\left(\overrightarrow{q_{1}}, \overrightarrow{0} ; \vec{q}\right)=\mathcal{K}_{2}\left(\overrightarrow{q_{1}}, \vec{q} ; \vec{q}\right)=\mathcal{K}_{3}\left(\vec{q}_{1}, \overrightarrow{0} ; \vec{q}\right)=\mathcal{K}_{3}(\vec{q}, \vec{q} ; \vec{q})=0,
$$

we can present in this limit the bootstrap condition $(2.8)$ in the following form:

$$
\begin{aligned}
& \frac{\left(\vec{q}^{2}\right)^{-2 \epsilon}}{\pi^{1+\epsilon} \Gamma(1-\epsilon)} \int \frac{d^{D-2} q_{2}}{\vec{q}_{2}^{2} \vec{q}_{2}^{\prime 2}}\left[\mathcal{K}_{1}+\mathcal{K}_{2}+\mathcal{K}_{3}+f_{B}\left(-\frac{11}{6} \ln \left(\frac{\vec{q}_{2}^{2} \vec{q}_{2}^{\prime 2}}{\vec{q}_{1}^{2} \vec{q}_{1}^{2}}\right)+\frac{1}{2} \ln \left(\frac{\vec{q}_{1}^{2}}{\vec{q}^{2}}\right) \ln \left(\frac{\vec{q}_{1}^{\prime 2}}{\vec{q}^{2}}\right)\right.\right. \\
& \left.\left.-\frac{1}{2} \ln \left(\frac{\vec{q}_{2}^{2}}{\vec{q}^{2}}\right) \ln \left(\frac{\vec{q}_{2}^{\prime 2}}{\vec{q}^{2}}\right)\right)\right]=-\frac{1}{\epsilon^{2}}\left[\frac{11}{3}+\left(2 \psi^{\prime}(1)-\frac{67}{9}\right) \epsilon+\left(\frac{404}{27}+\psi^{\prime \prime}(1)-\frac{22}{3} \psi^{\prime}(1)\right) \epsilon^{2}\right] \\
& -\frac{2}{\epsilon}\left[\frac{11}{3}+\left(2 \psi^{\prime}(1)-\frac{67}{9}\right) \epsilon\right] \ln \left(\frac{\vec{q}_{1}^{2} \vec{q}_{1}^{\prime 2}}{\left(\vec{q}^{2}\right)^{2}}\right)-\frac{22}{3}\left(\ln ^{2}\left(\frac{\vec{q}_{1}^{2}}{\vec{q}^{2}}\right)+\ln ^{2}\left(\frac{\vec{q}_{1}^{\prime 2}}{\vec{q}^{2}}\right)\right) .
\end{aligned}
$$

It is easy to see that due to (2.16) the only divergent integral here is that of $\mathcal{K}_{1}$. The divergent part of this integral should cancel the terms of order $1 / \epsilon$ and $1 / \epsilon^{2}$ which appear explicitly in the R.H.S. of (2.17). Indeed, we have (for details of the calculation see Appendix A) in the limit $\epsilon \rightarrow 0$

$$
\frac{\left(\vec{q}^{2}\right)^{-2 \epsilon}}{\pi^{1+\epsilon} \Gamma(1-\epsilon)} \int \frac{d^{D-2} q_{2}}{\vec{q}_{2}^{2} \vec{q}_{2}^{\prime 2}} f_{B} \frac{\left(\vec{k}^{2}\right)^{\epsilon}}{\epsilon}=\frac{1}{\epsilon^{2}}\left[1+2 \epsilon \ln \left(\frac{\vec{q}_{1}^{2} \vec{q}_{1}^{\prime 2}}{\left(\vec{q}^{2}\right)^{2}}\right)\right.
$$




$$
\left.+\epsilon^{2}\left(2 \ln ^{2}\left(\frac{\vec{q}_{1}^{2}}{\vec{q}^{2}}\right)+2 \ln ^{2}\left(\frac{\vec{q}_{1}^{\prime 2}}{\vec{q}^{2}}\right)+\ln \left(\frac{\vec{q}_{1}^{2}}{\vec{q}^{2}}\right) \ln \left(\frac{\vec{q}_{1}^{2}}{\vec{q}^{2}}\right)-\psi^{\prime}(1)\right)\right],
$$

and therefore (2.17) takes the form

$$
\begin{gathered}
\frac{\left(\vec{q}^{2}\right)^{-\epsilon}}{\pi^{1+\epsilon} \Gamma(1-\epsilon)} \int \frac{d^{D-2} q_{2}}{\vec{q}_{2}^{2} \vec{q}_{2}^{\prime 2}}\left[\mathcal{K}_{2}+\mathcal{K}_{3}+f_{B}\left(-\frac{11}{6} \ln \left(\frac{\vec{q}_{2}^{2} \vec{q}_{2}^{\prime 2}}{\vec{q}_{1}^{2} \vec{q}_{1}^{\prime 2}}\right)+\frac{1}{2} \ln \left(\frac{\vec{q}_{1}^{2}}{\vec{q}^{2}}\right) \ln \left(\frac{\vec{q}_{1}^{\prime 2}}{\vec{q}^{2}}\right)\right.\right. \\
\left.\left.-\frac{1}{2} \ln \left(\frac{\vec{q}_{2}^{2}}{\vec{q}^{2}}\right) \ln \left(\frac{\vec{q}_{2}^{2}}{\vec{q}^{2}}\right)\right)\right]=6 \psi^{\prime \prime}(1)+\frac{11}{3} \ln \left(\frac{\vec{q}_{1}^{2}}{\vec{q}^{2}}\right) \ln \left(\frac{\vec{q}_{1}^{\prime 2}}{\vec{q}^{2}}\right) .
\end{gathered}
$$

Although due to (2.16) the integrals in the L.H.S. of (2.19) are finite at $\epsilon \rightarrow 0$, we retain the dimensional regularization, since separate terms in them are still divergent. Using known integrals

$$
\begin{aligned}
I_{1}(\vec{q}) & =\frac{\left(\vec{q}^{2}\right)^{1-\epsilon}}{\pi^{1+\epsilon} \Gamma(1-\epsilon)} \int d^{D-2} q_{2} \frac{1}{\vec{q}_{2}^{2}\left(\vec{q}_{2}-\vec{q}\right)^{2}}=\frac{\Gamma^{2}(\epsilon)}{\Gamma(2 \epsilon)}=\frac{2}{\epsilon}+O(\epsilon) \\
I_{2}(\vec{q}) & =\frac{\left(\vec{q}^{2}\right)^{1-\epsilon}}{\pi^{1+\epsilon} \Gamma(1-\epsilon)} \int d^{D-2} q_{2} \frac{\ln \left(\vec{q}_{2}^{2} / \vec{q}^{2}\right)}{\vec{q}_{2}^{2}\left(\vec{q}_{2}-\vec{q}\right)^{2}} \\
& =\frac{\Gamma^{2}(\epsilon)}{\Gamma(2 \epsilon)}[\psi(\epsilon)-\psi(2 \epsilon)+\psi(1)-\psi(1-\epsilon)]=-\frac{1}{\epsilon^{2}}+\psi^{\prime}(1)+O(\epsilon) \\
I_{3}(\vec{q}) & =\frac{\left(\vec{q}^{2}\right)^{1-\epsilon}}{\pi^{1+\epsilon} \Gamma(1-\epsilon)} \int d^{D-2} q_{2} \frac{\ln ^{2}\left(\vec{q}_{2}^{2} / \vec{q}^{2}\right)}{\vec{q}_{2}^{2}\left(\vec{q}_{2}-\vec{q}\right)^{2}} \\
& =\frac{\Gamma^{2}(\epsilon)}{\Gamma(2 \epsilon)}\left[(\psi(\epsilon)-\psi(2 \epsilon)+\psi(1)-\psi(1-\epsilon))^{2}+\psi^{\prime}(\epsilon)-\psi^{\prime}(2 \epsilon)\right. \\
& \left.-\psi^{\prime}(1)+\psi(1-\epsilon)\right]=\frac{2}{\epsilon^{3}}-\frac{2 \psi^{\prime}(1)}{\epsilon}-2 \psi^{\prime \prime}(1)+O(\epsilon), \\
I_{4}(\vec{q}) & =\frac{\left(\vec{q}^{2}\right)^{1-\epsilon}}{\pi^{1+\epsilon} \Gamma(1-\epsilon)} \int d^{D-2} q_{2} \frac{\ln \left(\vec{q}_{2}^{2} / \vec{q}^{2}\right) \ln \left(\left(\vec{q}_{2}-\vec{q}\right)^{2} / \vec{q}^{2}\right)}{\vec{q}_{2}^{2}\left(\vec{q}_{2}-\vec{q}\right)^{2}} \\
& =\frac{\Gamma^{2}(\epsilon)}{\Gamma(2 \epsilon)}\left[(\psi(\epsilon)-\psi(2 \epsilon)+\psi(1)-\psi(1-\epsilon))^{2}+\psi^{\prime}(1-\epsilon)-\psi^{\prime}(2 \epsilon)\right] \\
& =-2 \psi^{\prime \prime}(1)+O(\epsilon),
\end{aligned}
$$

which can be easily calculated with the help of the generalized Feynman parametrization (see Eq. (A.1) in Appendix A), and (2.15), we obtain the bootstrap condition in the form:

$$
\begin{array}{r}
{\left[\left\{-3 \psi^{\prime \prime}(1)+\frac{1}{24} \ln ^{3}\left(\frac{\vec{q}_{1}^{2}}{\vec{q}^{2}}\right)-\frac{1}{4} \ln ^{2}\left(\frac{\vec{q}_{1}^{2}}{\vec{q}^{2}}\right) \ln \left(\frac{\vec{q}_{1}^{\prime 2}}{\vec{q}^{2}}\right)-\frac{1}{2} I\left(\vec{q}_{1}^{\prime 2}, \vec{q}^{2} ; \vec{q}_{1}^{2}\right)\right.\right.} \\
\left.\left.+\frac{1}{2} I\left(\vec{q}^{2}, \vec{q}_{1}^{2} ; \vec{q}_{1}^{\prime 2}\right)-\frac{3}{4} J\left(\vec{q}_{1}^{2}, \vec{q}^{2} ; \vec{q}_{1}^{\prime 2}\right)\right\}+\left\{\vec{q}_{1} \leftrightarrow \vec{q}_{1}^{\prime}\right\}\right]+\frac{1}{\pi} \int \frac{d \vec{q}_{2}}{\vec{q}_{2}^{2} \vec{q}_{2}^{\prime 2}} \mathcal{K}_{3}=0,
\end{array}
$$

where

$$
\begin{gathered}
I\left(\vec{p}_{1}^{2}, \vec{p}_{2}^{2} ;\left(\vec{p}_{1}-\vec{p}_{2}\right)^{2}\right)=\frac{\left(\vec{p}_{1}-\vec{p}_{2}\right)^{2}}{\pi} \int d \vec{p} \frac{\ln \left(\vec{p}_{2}^{2} / \vec{p}^{2}\right) \ln \left(\left(\vec{p}_{1}-\vec{p}_{2}\right)^{2} /\left(\vec{p}-\vec{p}_{2}\right)^{2}\right)}{\left(\vec{p}-\vec{p}_{1}\right)^{2}\left(\vec{p}-\vec{p}_{2}\right)^{2}} \\
J\left(\vec{p}_{1}^{2}, \vec{p}_{2}^{2} ;\left(\vec{p}_{1}-\vec{p}_{2}\right)^{2}\right)=\frac{\left(\vec{p}_{1}-\vec{p}_{2}\right)^{2}}{\pi} \int d \vec{p} \frac{\ln \left(\vec{p}_{1}^{2} / \vec{p}^{2}\right) \ln \left(\vec{p}_{2}^{2} / \vec{p}^{2}\right)}{\left(\vec{p}-\vec{p}_{1}\right)^{2}\left(\vec{p}-\vec{p}_{2}\right)^{2}}
\end{gathered}
$$

and $\mathcal{K}_{3}$ is defined in (2.14). 


\section{Proof of the fulfillment of the bootstrap condition}

Although all integrals in (2.24) can in principle be calculated analytically, arising expressions are so long and cumbersome that we restrict ourselves to present them in terms of onedimensional integrals. The derivation of such representations for the integrals $I$ and $J$ defined in Eqs. (2.25) and (2.26) is given in Appendix A and for the integral with $\mathcal{K}_{3}$ in Appendix B.

Moreover, the proof of the bootstrap relation can be simplified in the following way. The integrals entering the bootstrap condition, as well as explicitly presented logarithms, are functions of three variables: $q_{1}^{2} \equiv-\vec{q}_{1}^{2}, q_{1}^{\prime 2} \equiv-\vec{q}_{1}^{\prime 2}$ and $q^{2} \equiv-\vec{q}^{2}$. Let us fix $q_{1}^{2} \leq 0, q_{1}^{\prime 2} \leq 0$ and consider the dependence from $q^{2}$. All integrals entering the bootstrap condition (2.24), as well as explicit logarithms presented there, are real analytical functions of $q^{2}$ with the cut $0 \leq q^{2}<\infty$. They are determined, up to a constant (from the point of view of dependence from $q^{2}$; but it still can be a function of $q_{1}^{2}$ and $q_{1}^{\prime 2}$ ), by their discontinuities on the cut. The calculation of the discontinuities on the cut is a much easier problem than the calculation of the whole functions; therefore, let us prove the bootstrap relation for the discontinuities. If we manage to do it, the only thing which will remain to be done is to prove the bootstrap at some fixed point in the $q^{2}$ plane. Taking this point at $\infty$, we again simplify the calculations drastically.

Let start with the discontinuities. For real functions they are equal to their imaginary parts on the upper edge of the cut multiplied by $2 i$. The values of the functions on the upper edge of the cut are given by the expressions obtained for $q^{2}=-\vec{q}^{2} \leq 0$ with the substitution $\vec{q}^{2} \rightarrow-q^{2}-i 0$. Therefore for the logarithmic functions explicitly presented in (2.24) the calculation of the discontinuities is trivial. For the integrals $I$ is easy to obtain from the representations given in Appendix A:

$$
\begin{gathered}
\frac{1}{\pi} \Im I\left(\vec{q}_{1}^{2},-q^{2}-i 0 ; \vec{q}_{1}^{\prime 2}\right)=2 \psi^{\prime}(1)+\frac{1}{2} \ln \left(\frac{1-a}{a}\right) \ln (a(1-a))+\operatorname{Li}_{2}(a)-\operatorname{Li}_{2}(1-a) \\
+\int_{0}^{a} \frac{d x}{x(1-x)} \ln \left(1-\frac{x}{a}+\frac{x(1-x) \vec{q}_{1}^{\prime 2}}{q^{2}}\right) \\
\frac{1}{\pi} \Im I\left(-q^{2}-i 0, \vec{q}_{1}^{2} ; \vec{q}_{1}^{\prime 2}\right)=\psi^{\prime}(1)+\ln \left(\frac{1-a}{a}\right) \ln (1-a)+\operatorname{Li}_{2}(a)-\operatorname{Li}_{2}(1-a) \\
+\int_{0}^{a} \frac{d x}{x(1-x)} \ln \left(1-\frac{x}{a}+\frac{x(1-x) \vec{q}_{1}^{\prime 2}}{q^{2}}\right)
\end{gathered}
$$

where

$$
a=\frac{q^{2}}{\vec{q}_{1}^{2}+q^{2}}, \quad \operatorname{Li}_{2}(z)=-\int_{0}^{z} \frac{d x}{x} \ln (1-x) .
$$

The bootstrap relation contains these integrals in the combination for which the imaginary part is quite simple:

$$
-\frac{1}{2} \frac{1}{\pi} \Im I\left(\vec{q}_{1}^{2},-q^{2}-i 0 ; \vec{q}_{1}^{\prime 2}\right)+\frac{1}{2} \frac{1}{\pi} \Im I\left(-q^{2}-i 0, \vec{q}_{1}^{2} ; \vec{q}_{1}^{\prime 2}\right)=-\frac{\psi^{\prime}(1)}{2}+\frac{1}{4} \ln ^{2}\left(\frac{\vec{q}_{1}^{2}}{q^{2}}\right) .
$$


For the integral $J$ the representation of Appendix A gives:

$$
\begin{gathered}
\frac{1}{\pi} \Im J\left(\vec{q}_{1}^{2},-q^{2}-i 0 ; \vec{q}_{1}^{\prime 2}\right)=2 \psi^{\prime}(1)+\frac{1}{2} \ln \left(\frac{1-a}{a}\right) \ln (a(1-a))+\operatorname{Li}_{2}(a)-\operatorname{Li}_{2}(1-a) \\
-2 \operatorname{Li}_{2}\left(\frac{-a}{1-a}\right)+2 \int_{0}^{a} \frac{d x}{x(1-x)} \ln \left(1-\frac{x}{a}+\frac{x(1-x) \vec{q}_{1}^{\prime 2}}{q^{2}}\right) .
\end{gathered}
$$

The integral terms in (3.31) give dilogarithms with rather complicated arguments:

$$
\begin{gathered}
\int_{0}^{a} \frac{d x}{x(1-x)} \ln \left(1-\frac{x}{a}+\frac{x(1-x) \vec{q}_{1}^{\prime 2}}{q^{2}}\right)=-2 \operatorname{Li}_{2}\left(\frac{\kappa^{+}-\vec{q}_{1}^{2}}{q^{2}+\vec{q}_{1}^{2}}\right)-2 \operatorname{Li}_{2}\left(\frac{\kappa^{-}-\vec{q}_{1}^{\prime 2}}{q^{2}+\vec{q}_{1}^{2}}\right) \\
-\operatorname{Li}_{2}\left(\frac{\vec{q}_{1}^{\prime 2}}{\kappa^{+}}\right)+\operatorname{Li}_{2}\left(\frac{\vec{q}_{1}^{2}}{\kappa^{+}}\right)+\ln \left(\frac{\kappa^{+}-\vec{q}_{1}^{\prime 2}}{q^{2}}\right) \ln \left(\frac{\kappa^{+}}{\kappa^{-}}\right)+\frac{1}{2} \ln ^{2}\left(\frac{\kappa^{+}}{q^{2}}\right) \\
-\ln \left(\frac{\kappa^{+}}{q^{2}}\right) \ln \left(\frac{\vec{q}_{1}^{2} \vec{q}_{1}^{\prime 2}}{\left(q^{2}\right)^{2}}\right)-2 \ln ^{2}\left(\frac{q^{2}+\vec{q}_{1}^{2}}{q^{2}}\right)+2 \ln \left(\frac{q^{2}+\vec{q}_{1}^{2}}{q^{2}}\right) \ln \left(\frac{\vec{q}_{1}^{2} \vec{q}_{1}^{\prime 2}}{\left(q^{2}\right)^{2}}\right)-\frac{1}{2} \ln ^{2}\left(\frac{\vec{q}_{1}^{2}}{q^{2}}\right),
\end{gathered}
$$

where

$$
\kappa^{ \pm}=\frac{1}{2}\left(q^{2}+\vec{q}_{1}^{2}+\vec{q}_{1}^{\prime 2} \pm \sqrt{\left(q^{2}+\vec{q}_{1}^{2}+\vec{q}_{1}^{\prime 2}\right)^{2}-4 \vec{q}_{1}^{2} \vec{q}_{1}^{\prime 2}}\right) .
$$

But their sum with the terms $\left(\vec{q}_{1} \leftrightarrow \vec{q}_{1}^{\prime}\right)$, with account of (C.12), drastically simplifies:

$$
\begin{array}{r}
\int_{0}^{a} \frac{d x}{x(1-x)} \ln \left(1-\frac{x}{a}+\frac{x(1-x) \vec{q}_{1}^{\prime 2}}{q^{2}}\right)+\left(\vec{q}_{1}^{2} \leftrightarrow \vec{q}_{1}^{\prime 2}\right)=2 \operatorname{Li}_{2}(1-a) \\
-\frac{1}{2} \ln \left(\frac{\kappa^{-}}{q^{2}}\right) \ln \left(\frac{\kappa^{+}}{q^{2}}\right)-2 \psi^{\prime}(1)+\ln ^{2}(a)-\frac{1}{2} \ln ^{2}\left(\frac{1-a}{a}\right)+\left(\vec{q}_{1}^{2} \leftrightarrow \vec{q}_{1}^{\prime 2}\right) .
\end{array}
$$

Using this relation and the identity

$$
\mathrm{Li}_{2}(a)+3 \mathrm{Li}_{2}(1-a)-2 \mathrm{Li}_{2}\left(\frac{-a}{1-a}\right)=3 \psi^{\prime}(1)+\ln \left(\frac{1-a}{a}\right) \ln \left(\frac{1-a}{a^{2}}\right)-2 \ln ^{2}(a),
$$

which one can easily check comparing both sides of the identity at $a=0$ and their derivatives with respect to $a$, we obtain from (3.31)

$$
\begin{gathered}
\frac{1}{\pi} \Im J\left(\vec{q}_{1}^{2},-q^{2}-i 0 ; \vec{q}_{1}^{2}\right)+\left(\vec{q}_{1}^{2} \leftrightarrow \vec{q}_{1}^{\prime 2}\right) \\
=-\ln \left(\frac{\kappa^{-}}{q^{2}}\right) \ln \left(\frac{\kappa^{+}}{q^{2}}\right)+\frac{1}{2} \ln ^{2}\left(\frac{\vec{q}_{1}^{2}}{q^{2}}\right)+\psi^{\prime}(1)+\left(\vec{q}_{1}^{2} \leftrightarrow \vec{q}_{1}^{\prime 2}\right) .
\end{gathered}
$$

The discontinuity of the term with $\mathcal{K}_{3}$ in (2.24) can be calculated using the representations obtained in Appendix B. But it seems that the easiest way to find it is to rewrite the integral with $\mathcal{K}_{3}$ in (2.24) in the Minkowski space and to take the discontinuity according to Cutkosky rules. This way is described in Appendix C. In any case, the result is

$$
\frac{\Im A}{\pi}=-\frac{3}{2} \ln \left(\frac{\kappa^{-}}{q^{2}}\right) \ln \left(\frac{\kappa^{+}}{q^{2}}\right)+\frac{1}{4} \ln ^{2}\left(\frac{\vec{q}_{1}^{2} \vec{q}_{1}^{\prime 2}}{\left(q^{2}\right)^{2}}\right)+\frac{1}{2} \ln \left(\frac{\vec{q}_{1}^{2}}{q^{2}}\right) \ln \left(\frac{\vec{q}_{1}^{\prime 2}}{q^{2}}\right) .
$$


Here $A$ is the analytic continuation (see (C.2)) of the term with $\mathcal{K}_{3}$ in the bootstrap relation (2.24).

The relation imposed by (2.24) on the imaginary parts is:

$$
\begin{gathered}
\left\{\left[-\frac{1}{8} \ln ^{2}\left(\frac{\vec{q}_{1}^{2}}{q^{2}}\right)+\frac{5 \pi^{2}}{24}-\frac{1}{2} \ln \left(\frac{\vec{q}_{1}^{2}}{q^{2}}\right) \ln \left(\frac{\vec{q}_{1}^{\prime 2}}{q^{2}}\right)-\frac{1}{2} \frac{1}{\pi} \Im I\left(\vec{q}_{1}^{2},-q^{2}-i 0 ; \vec{q}_{1}^{\prime 2}\right)\right.\right. \\
\left.\left.+\frac{1}{2} \frac{1}{\pi} \Im I\left(-q^{2}-i 0, \vec{q}_{1}^{2} ; \vec{q}_{1}^{\prime 2}\right)-\frac{3}{4} \frac{1}{\pi} \Im J\left(\vec{q}_{1}^{2},-q^{2}-i 0 ; \vec{q}_{1}^{\prime 2}\right)\right]+\left[\vec{q}_{1}^{2} \leftrightarrow \vec{q}_{1}^{\prime 2}\right]\right\}+\frac{\Im A}{\pi}=0 .
\end{gathered}
$$

Using (3.34), (3.36) and (3.37) it is easy to see that this relation is satisfied.

Now in order to complete the proof of fulfillment of the bootstrap condition it is sufficient to prove that it is satisfied in the limit $\vec{q}^{2} \gg \vec{q}_{1}^{2}, \vec{q}^{2} \gg \vec{q}_{1}^{\prime 2}$. From the expressions for the integrals $I$ and $J$ obtained in Appendix A we obtain in this limit

$$
\begin{gathered}
I\left(\vec{q}_{1}^{2}, \vec{q}^{2} ; \vec{q}_{1}^{\prime 2}\right) \simeq-\zeta(2) \ln \left(\frac{\vec{q}^{2}}{\vec{q}_{1}^{2}}\right)+2 \zeta(3), \\
I\left(\vec{q}^{2}, \vec{q}_{1}^{2} ; \vec{q}_{1}^{\prime 2}\right) \simeq-\frac{1}{6} \ln ^{3}\left(\frac{\vec{q}^{2}}{\vec{q}_{1}^{2}}\right)-\zeta(2) \ln \left(\frac{\vec{q}^{2}}{\vec{q}_{1}^{2}}\right)+2 \zeta(3),
\end{gathered}
$$

and

$$
J\left(\vec{q}_{1}^{2}, \vec{q}^{2} ; \vec{q}_{1}^{2}\right) \simeq-\frac{1}{6} \ln ^{3}\left(\frac{\vec{q}^{2}}{\vec{q}_{1}^{2}}\right)-2 \zeta(2) \ln \left(\frac{\vec{q}^{2}}{\vec{q}_{1}^{2}}\right)+4 \zeta(3),
$$

where $\zeta(n)$ is the Riemann zeta-function, $\zeta(2)=\psi^{\prime}(1)=\pi^{2} / 6,2 \zeta(3)=-\psi^{\prime \prime}(1)$.

The value of the contribution of the term with $\mathcal{K}_{3}$ in this limit is found in Appendix B:

$$
\begin{gathered}
\frac{1}{\pi} \int \frac{d \vec{q}_{2}}{\vec{q}_{2}^{2} \vec{q}_{2}^{\prime 2}} \mathcal{K}_{3} \simeq-\frac{1}{4} \ln \left(\frac{\vec{q}^{2}}{\vec{q}_{1}^{\prime 2}}\right) \ln \left(\frac{\vec{q}^{2}}{\vec{q}_{1}^{2}}\right)\left(\ln \left(\frac{\vec{q}^{2}}{\vec{q}_{1}^{2}}\right)+\ln \left(\frac{\vec{q}^{2}}{\vec{q}_{1}^{\prime 2}}\right)\right) \\
-\frac{3 \zeta(2)}{2}\left(\ln \left(\frac{\vec{q}^{2}}{\vec{q}_{1}^{2}}\right)+\ln \left(\frac{\vec{q}^{2}}{\vec{q}_{1}^{\prime 2}}\right)\right)-6 \zeta(3) .
\end{gathered}
$$

Putting the expressions (3.39)-(3.42) into the equation (2.24) one can easily see that it is satisfied in the limit of large $\vec{q}^{2}$.

This completes the proof of the strong bootstrap condition for the NLO color octet kernel.

\section{Discussion}

The BFKL approach to the description of high energy processes is based on the gluon Reggeization. More precisely, in the derivation of the representation for scattering amplitudes and of the BFKL equation, the Reggeized form for the amplitudes with the gluon quantum numbers in the $t$-channels was assumed. This assumption was proved in the leading logarithmic approximation [14], but still remains a hypothesis beyond this approximation. 
Now a lot of results in the BFKL approach are obtained in the next-to-leading order for the kernel of the BFKL equation [2, 3, 4, 5, 6] and the impact factors [7, 8, 9, 10, 11, 12, 13. These results are widely discussed in the literature. Therefore it is very desirable to prove the hypothesis of the gluon Reggeization in the NLO or, at least, to check it as carefully as possible.

The self-consistency of the hypothesis demands the fulfillment of bootstrap conditions arising from the requirement that amplitudes obtained in the BFKL approach by use of the BFKL equation in the antisymmetric color octet state must have the Reggeized form which was assumed in the derivation. Now the fulfillment of the NLO bootstrap conditions for the elastic scattering amplitudes [四] is proved [5, 7, 8, 15].

The bootstrap conditions must be satisfied for all amplitudes involved into the derivation of the BFKL equation, i.e. for the amplitudes of production of any number of particles in the multi-Regge and quasi multi-Regge kinematics. The so called strong bootstrap conditions suggested without derivation in [18, 19] appear among them [17]. Although the conditions for the elastic amplitudes are already very restrictive, so that their fulfillment can convince about the correctness of the hypothesis of Reggeization, the conditions for inelastic amplitudes are even stronger.

In this paper we have completed the proof of the strong bootstrap conditions. The bootstrap condition proved to be satisfied is so strong that without the Reggeization its fulfillment would seem a miracle. Although the Reggeized form for inelastic amplitudes implies another set of conditions [17], which are not yet proved, the fulfillment of the strong bootstrap heavily supports the conclusion that also these conditions are satisfied. Moreover, the fulfillment of these conditions has to give the possibility to prove the hypothesis of gluon Reggeization in the NLO.

Acknowledgment: V.S.F. thanks the Alexander von Humboldt foundation for the research award, the Universität Hamburg and DESY for their warm hospitality while a part of this work was done. F. Caporale (Università della Calabria and Istituto Nazionale di Fisica Nucleare, Gruppo collegato di Cosenza) participated to the early stages of the present work. 


\section{A Appendix}

In this Appendix we give some details about the calculation of the integral in the L.H.S. of Eq. (2.18) and of the integrals $I\left(\vec{p}_{1}^{2}, \vec{p}_{2}^{2} ;\left(\vec{p}_{1}-\vec{p}_{2}\right)^{2}\right)$ and $J\left(\vec{p}_{1}^{2}, \vec{p}_{2}^{2} ;\left(\vec{p}_{1}-\vec{p}_{2}\right)^{2}\right)$ defined in $(2.25)$ and (2.26), respectively. The first of these three integrals will be calculated analytically in the $\epsilon$-expansion. The other two will instead be presented in terms of one-dimensional integrals, since they can not be expressed in terms of elementary functions, and their expressions in terms of known dilogarithms would be long and cumbersome.

A necessary ingredient in all three cases will be the generalized Feynman parametrization, which we recall here for the convenience:

$$
\frac{1}{a_{1}^{\alpha_{1}} \ldots a_{n}^{\alpha_{n}}}=\frac{\Gamma\left(\sum_{i} \alpha_{i}\right)}{\Gamma\left(\alpha_{1}\right) \ldots \Gamma\left(\alpha_{n}\right)} \int_{0}^{1} \ldots \int_{0}^{1} \frac{d x_{1} \ldots d x_{n} \delta\left(1-\sum_{i} x_{i}\right) x_{1}^{\alpha_{1}-1} \ldots x_{n}^{\alpha_{n}-1}}{\left(\sum_{i} a_{i} x_{i}\right)^{\sum_{i} \alpha_{i}}} .
$$

Let us start from the integral in (2.18), related to the contribution of $\mathcal{K}_{1}$ to the bootstrap condition (2.17),

$$
\begin{gathered}
I_{\mathcal{K}_{1}} \equiv \frac{\left(\vec{q}^{2}\right)^{-2 \epsilon}}{\pi^{1+\epsilon} \Gamma(1-\epsilon)} \int \frac{d^{D-2} q_{2}}{\vec{q}_{2}^{2} \vec{q}_{2}^{\prime 2}} f_{B} \frac{\left(\vec{k}^{2}\right)^{\epsilon}}{\epsilon} \\
=\frac{\left(\vec{q}^{2}\right)^{-2 \epsilon}}{\pi^{1+\epsilon} \Gamma(1-\epsilon)} \int \frac{d^{D-2} q_{2}}{\vec{q}_{2}^{2} \vec{q}_{2}^{\prime 2}}\left(\frac{\vec{q}_{1}^{2} \vec{q}_{2}^{\prime 2}+\vec{q}_{2}^{2} \vec{q}_{1}^{\prime 2}}{\left(\vec{q}_{1}-\vec{q}_{2}\right)^{2}}-\vec{q}^{2}\right) \frac{\left[\left(\vec{q}_{1}-\vec{q}_{2}\right)^{2}\right]^{\epsilon}}{\epsilon}
\end{gathered}
$$

where the definition of $f_{B}$, given in (1.6), and $\vec{k}=\vec{q}_{1}-\vec{q}_{2}$ have been used. We need to calculate $I_{\mathcal{K}_{1}}$ with accuracy up to order $\epsilon^{0}$. First of all, $I_{\mathcal{K}_{1}}$ can be split into the sum of three contributions:

$$
\begin{aligned}
I_{\mathcal{K}_{1}} & =\frac{1}{\pi^{1+\epsilon} \Gamma(1-\epsilon)} \frac{\left(\vec{q}^{2}\right)^{-2 \epsilon} \vec{q}_{1}^{2}}{\epsilon} \int \frac{d^{D-2} q_{2}}{\vec{q}_{2}^{2}\left[\left(\vec{q}_{2}-\vec{q}_{1}\right)^{2}\right]^{1-\epsilon}} \\
& +\frac{1}{\pi^{1+\epsilon} \Gamma(1-\epsilon)} \frac{\left(\vec{q}^{2}\right)^{-2 \epsilon} \vec{q}_{1}^{\prime 2}}{\epsilon} \int \frac{d^{D-2} q_{2}}{{\overrightarrow{q_{2}^{\prime}}}^{2}\left[\left(\vec{q}_{2}-\vec{q}_{1}\right)^{2}\right]^{1-\epsilon}} \\
& -\frac{1}{\pi^{1+\epsilon} \Gamma(1-\epsilon)} \frac{\left(\vec{q}^{2}\right)^{1-2 \epsilon}}{\epsilon} \int \frac{d^{D-2} q_{2}}{\vec{q}_{2}^{2} \vec{q}_{2}^{\prime 2}\left[\left(\vec{q}_{2}-\vec{q}_{1}\right)^{2}\right]^{-\epsilon}} \\
& \equiv I_{\mathcal{K}_{1}}^{(a)}\left(\vec{q}_{1}\right)+I_{\mathcal{K}_{1}}^{(a)}\left(\vec{q}_{1}^{\prime}\right)-I_{\mathcal{K}_{1}}^{(b)},
\end{aligned}
$$

with obvious notation. The integral $I_{\mathcal{K}_{1}}^{(a)}\left(\vec{q}_{1}\right)$ can by easily calculated for arbitrary $\epsilon$ through Feynman parametrization and by use of

$$
\int \frac{d^{D-2} k}{(2 \pi)^{D-1}} \frac{1}{\left(\vec{k}^{2}-2 \vec{k} \cdot \vec{p}+m^{2}\right)^{\alpha}}=\frac{2}{(4 \pi)^{D / 2}} \frac{\Gamma(\alpha+1-D / 2)}{\Gamma(\alpha)} \frac{1}{\left(m^{2}-\vec{p}^{2}\right)^{\alpha+1-D / 2}},
$$

giving

$$
I_{\mathcal{K}_{1}}^{(a)}\left(\vec{q}_{1}\right)=\frac{1}{\epsilon} \frac{\Gamma(1-2 \epsilon)}{\Gamma^{2}(1-\epsilon)} \frac{\Gamma(\epsilon) \Gamma(2 \epsilon)}{\Gamma(3 \epsilon)}\left(\frac{\vec{q}_{1}^{2}}{\vec{q}^{2}}\right)^{2 \epsilon}
$$


The integral in $I_{\mathcal{K}_{1}}^{(b)}$ is less trivial than the previous one, since it contains three factors in the denominator. Moreover, in the expression for $I_{\mathcal{K}_{1}}^{(b)}$ the parameter $\epsilon$ appears both explicitly (in the integrand and in the pre-factors) and implicitly, through $D$, in the integration measure over $\vec{q}_{2}$. This creates a problem: for the convergence of the integral over $q_{2}$ the real part of $\epsilon=(D-4) / 2$ has to be positive, whereas for the convergence of the integrals in the Feynman parametrization (A.1) with one of $\alpha$-s equal to $-\epsilon$ we have to suppose that $\epsilon$ has a negative real part. To escape this contradiction let us consider

$$
I_{\mathcal{K}_{1}}^{(b)}=\frac{1}{\pi^{1+\epsilon} \Gamma(1-\epsilon)} \frac{\left(\vec{q}^{2}\right)^{1-2 \epsilon}}{\epsilon} \int \frac{d^{D-2} q_{2}}{{\overrightarrow{q_{2}}}_{2}^{2} \vec{q}_{2}^{\prime 2}\left[\left(\vec{q}_{2}-\vec{q}_{1}\right)^{2}\right]^{-\epsilon_{1}}} .
$$

Since this integral is an analytic function of $\epsilon_{1}$ in the vicinity of $\epsilon_{1}=0$, we will calculate it supposing that the real part of $\epsilon_{1}$ is negative (and keeping $\left|\epsilon_{1}\right| \sim \epsilon$ ) and put $\epsilon_{1}=\epsilon$ after this.

The first step for the calculation of this integral is to apply the generalized Feynman parametrization (A.1) and to perform the integration over $\overrightarrow{q_{2}}$ by use of (A.4); this gives

$$
\begin{gathered}
I_{\mathcal{K}_{1}}^{(b)}=-\frac{\Gamma\left(1-\epsilon_{1}-\epsilon\right)}{\Gamma\left(1-\epsilon_{1}\right) \Gamma(1-\epsilon)} \frac{\epsilon_{1}}{\epsilon}\left(\vec{q}^{2}\right)^{1-2 \epsilon} \\
\times \int_{0}^{1} d x \int_{0}^{1} d y \frac{(1-y)^{\epsilon+\epsilon_{1}} y^{-1-\epsilon_{1}}}{\left[y\left(x \vec{q}_{1}^{\prime 2}+(1-x) \vec{q}_{1}^{2}\right)+x(1-x)(1-y) \vec{q}^{2}\right]^{1-\epsilon-\epsilon_{1}}} \\
=-\frac{\Gamma\left(1-\epsilon_{1}-\epsilon\right)}{\Gamma\left(1-\epsilon_{1}\right) \Gamma(1-\epsilon)} \frac{\epsilon_{1}}{\epsilon}\left(\vec{q}^{2}\right)^{1-2 \epsilon} \\
\times\left[\int_{0}^{1} d x \int_{0}^{1} d y \frac{\left[(1-y)^{\epsilon+\epsilon_{1}}-(1-y)^{-\epsilon}\right] y^{-1-\epsilon_{1}}}{\left[y\left(x \vec{q}_{1}^{\prime 2}+(1-x) \vec{q}_{1}^{2}\right)+x(1-x)(1-y) \vec{q}^{2}\right]^{1-\epsilon-\epsilon_{1}}}\right. \\
\left.+\int_{0}^{1} d x \int_{0}^{1} d y \frac{(1-y)^{-\epsilon} y^{-1-\epsilon_{1}}}{\left[y\left(x \vec{q}_{1}^{\prime 2}+(1-x) \vec{q}_{1}^{2}\right)+x(1-x)(1-y) \vec{q}^{2}\right]^{1-\epsilon-\epsilon_{1}}}\right] .
\end{gathered}
$$

The last equality was done for convenience because the first integral in square brackets is $O(\epsilon)$ and can be neglected, while in the second one, the integration over $y$ can be easily done by using backwards the Feynman parametrization (A.1):

$$
\begin{gathered}
\int_{0}^{1} d x \int_{0}^{1} d y \frac{(1-y)^{-\epsilon} y^{-1-\epsilon_{1}}}{\left[y\left(x \vec{q}_{1}^{\prime 2}+(1-x) \vec{q}_{1}^{2}\right)+x(1-x)(1-y) \vec{q}^{2}\right]^{1-\epsilon-\epsilon_{1}}} \\
=-\frac{1}{\epsilon_{1}} \frac{\Gamma\left(1-\epsilon_{1}\right) \Gamma(1-\epsilon)}{\Gamma\left(1-\epsilon-\epsilon_{1}\right)} \int_{0}^{1} d x \frac{\left[x \vec{q}_{1}^{2}+(1-x) \vec{q}_{1}^{2}\right]^{\epsilon_{1}}}{\left[x(1-x) \vec{q}^{2}\right]^{1-\epsilon}} .
\end{gathered}
$$

So, replacing back $\epsilon_{1}$ with $\epsilon$, we can write with $O\left(\epsilon^{0}\right)$ accuracy

$$
I_{\mathcal{K}_{1}}^{(b)}=\frac{\left(\vec{q}^{2}\right)^{1-2 \epsilon}}{\epsilon} \int_{0}^{1} d x \frac{\left[x \vec{q}_{1}^{\prime 2}+(1-x) \vec{q}_{1}^{2}\right]^{\epsilon}}{\left[x(1-x) \vec{q}^{2}\right]^{1-\epsilon}} .
$$

The one-dimensional integral in the previous expression is needed up to $O(\epsilon)$ and its calculation can be performed by splitting the integration region into three parts, (i) $0<x<\delta$, 
(ii) $\delta<x<1-\delta$ and (iii) $1-\delta<x<1$, and by calculating the three resulting integrals in the limit $\delta \rightarrow 0$. This can be done without any difficulty and leads finally to

$$
I_{\mathcal{K}_{1}}^{(b)}=\frac{1}{\epsilon}\left\{\frac{1}{\epsilon}\left[\left(\frac{\vec{q}_{1}^{2}}{\vec{q}^{2}}\right)^{\epsilon}+\left(\frac{\vec{q}_{1}^{2}}{\vec{q}^{2}}\right)^{\epsilon}\right]+\epsilon\left(\frac{1}{2} \ln ^{2} \frac{\vec{q}_{1}^{2}}{{\overrightarrow{q_{1}^{\prime}}}^{2}}-2 \psi^{\prime}(1)\right)\right\}+O(\epsilon) .
$$

Putting the results of (A.5) and (A.8) into (A.3), we obtain, with $O\left(\epsilon^{0}\right)$ accuracy,

$$
\begin{gathered}
I_{\mathcal{K}_{1}}=\frac{1}{\epsilon^{2}}\left[1+2 \epsilon \ln \left(\frac{\vec{q}_{1}^{2} \vec{q}_{1}^{\prime 2}}{\left(\vec{q}^{2}\right)^{2}}\right)\right. \\
\left.+\epsilon^{2}\left(2 \ln ^{2}\left(\frac{\vec{q}_{1}^{2}}{\vec{q}^{2}}\right)+2 \ln ^{2}\left(\frac{\vec{q}_{1}^{2}}{\vec{q}^{2}}\right)+\ln \left(\frac{\vec{q}_{1}^{2}}{\vec{q}^{2}}\right) \ln \left(\frac{\vec{q}_{1}^{\prime 2}}{\vec{q}^{2}}\right)-\psi^{\prime}(1)\right)\right] .
\end{gathered}
$$

Let us consider now the integral $I\left(\vec{p}_{1}^{2}, \vec{p}_{2}^{2} ;\left(\vec{p}_{1}-\vec{p}_{2}\right)^{2}\right)$ defined in $(2.25)$ :

$$
\begin{aligned}
I\left(\vec{p}_{1}^{2}, \vec{p}_{2}^{2} ;\left(\vec{p}_{1}-\vec{p}_{2}\right)^{2}\right) & =\frac{\left(\vec{p}_{1}-\vec{p}_{2}\right)^{2}}{\pi} \int d \vec{p} \frac{\ln \left(\vec{p}_{2}^{2} / \vec{p}^{2}\right) \ln \left(\left(\vec{p}_{1}-\vec{p}_{2}\right)^{2} /\left(\vec{p}-\vec{p}_{2}\right)^{2}\right)}{\left(\vec{p}-\vec{p}_{1}\right)^{2}\left(\vec{p}-\vec{p}_{2}\right)^{2}} \\
& =\frac{1}{\pi} \int d \vec{k} \frac{\ln \left(\vec{k}_{2}^{2} / \vec{k}^{2}\right) \ln \left(1 /\left(\vec{k}-\vec{k}_{2}\right)^{2}\right)}{\left(\vec{k}-\vec{k}_{1}\right)^{2}\left(\vec{k}-\vec{k}_{2}\right)^{2}} \equiv \tilde{I}\left(\vec{k}_{1}^{2}, \vec{k}_{2}^{2}\right)
\end{aligned}
$$

where we have performed the change of integration variable

$$
\vec{p} \longrightarrow\left|\vec{p}_{1}-\vec{p}_{2}\right| \vec{k}
$$

and defined

$$
\vec{k}_{1} \equiv \frac{\vec{p}_{1}}{\left|\vec{p}_{1}-\vec{p}_{2}\right|}, \quad \vec{k}_{2} \equiv \frac{\vec{p}_{2}}{\left|\vec{p}_{1}-\vec{p}_{2}\right|}, \quad\left(\left(\vec{k}_{1}-\vec{k}_{2}\right)^{2}=1\right)
$$

Although $\tilde{I}\left(\vec{k}_{1}^{2}, \vec{k}_{2}^{2}\right)$ is convergent, we introduce nevertheless dimensional regularization, since divergences will appear in intermediate steps of the calculation. We will consider therefore the integral

$$
\tilde{I}\left(\vec{k}_{1}^{2}, \vec{k}_{2}^{2}\right)=\frac{1}{\pi^{1+\epsilon} \Gamma(1-\epsilon)} \int d^{D-2} k \frac{\ln \left(\vec{k}_{2}^{2} / \vec{k}^{2}\right) \ln \left(1 /\left(\vec{k}-\vec{k}_{2}\right)^{2}\right)}{\left(\vec{k}-\vec{k}_{1}\right)^{2}\left(\vec{k}-\vec{k}_{2}\right)^{2}},
$$

and keep only terms up to $O\left(\epsilon^{0}\right)$. First of all, we rewrite $\tilde{I}$ in the following form:

$$
\tilde{I}\left(\vec{k}_{1}^{2}, \vec{k}_{2}^{2}\right)=\frac{1}{\pi^{1+\epsilon} \Gamma(1-\epsilon)} \frac{\partial}{\partial \alpha} \frac{\partial}{\partial \beta}\left(\int d^{D-2} k \frac{\left(\vec{k}_{2}^{2}\right)^{\beta}}{\left(\vec{k}-\vec{k}_{1}\right)^{2}\left[\left(\vec{k}-\vec{k}_{2}\right)^{2}\right]^{1+\alpha}\left(\vec{k}^{2}\right)^{\beta}}\right)_{\alpha=0, \beta=0}
$$

Then, after Feynman parametrization and integration over $\vec{k}$, we obtain

$$
\tilde{I}\left(\vec{k}_{1}^{2}, \vec{k}_{2}^{2}\right)=\frac{1}{\Gamma(1-\epsilon)} \frac{\partial}{\partial \alpha} \frac{\partial}{\partial \beta}\left(\frac{\Gamma(1+\alpha+\beta-\epsilon)}{\Gamma(1+\alpha) \Gamma(1+\beta)} \beta \int_{0}^{1} d x \int_{0}^{1} d y \frac{y^{\epsilon}}{1-y}\left(\frac{1-x}{D_{y}}\right)^{\alpha}\right.
$$




$$
\begin{aligned}
& \left.\times\left(\frac{(1-y) \vec{k}_{2}^{2}}{y D_{y}}\right)^{\beta} D_{y}^{\epsilon-1}\right)_{\alpha=0, \beta=0} \\
= & \frac{1}{\Gamma(1-\epsilon)} \frac{\partial}{\partial \beta}\left(\frac{\Gamma(1+\beta-\epsilon)}{\Gamma(1+\beta)} \beta \int_{0}^{1} d x \int_{0}^{1} d y \frac{y^{\epsilon}}{1-y}\left(\frac{(1-y) \vec{k}_{2}^{2}}{y D_{y}}\right)^{\beta} D_{y}^{\epsilon-1}\right. \\
& \left.\times\left[\psi(1+\beta-\epsilon)-\psi(1)-\ln \left(\frac{D_{y}}{1-x}\right)\right]\right)_{\beta=0},
\end{aligned}
$$

where $D_{y} \equiv(1-y)\left(x \vec{k}_{1}^{2}+(1-x) \vec{k}_{2}^{2}\right)+y x(1-x)$. Using the property

$$
\int_{0}^{1} d y(1-y)^{\beta-1} f(y)=\frac{f(1)}{\beta}+\int_{0}^{1} d y(1-y)^{\beta-1}[f(y)-f(1)]
$$

and performing the derivative with respect to $\beta$, we arrive at

$$
\begin{gathered}
\tilde{I}\left(\vec{k}_{1}^{2}, \vec{k}_{2}^{2}\right)=\int_{0}^{1} d x\left\{\left(\psi(1-\epsilon)-\psi(1)+\ln \left(\frac{\vec{k}_{2}^{2}}{D_{1}}\right)\right)\left(\psi(1-\epsilon)-\psi(1)-\ln \left(\frac{D_{1}}{1-x}\right)\right) D_{1}^{\epsilon-1}\right. \\
+\psi^{\prime}(1-\epsilon) D_{1}^{\epsilon-1}+\int_{0}^{1} \frac{d y}{1-y}\left[(\psi(1-\epsilon)-\psi(1))\left(y^{\epsilon} D_{y}^{\epsilon-1}-D_{1}^{\epsilon-1}\right)\right. \\
\left.\left.-\ln \left(\frac{D_{y}}{1-x}\right) y^{\epsilon} D_{y}^{\epsilon-1}+\ln \left(\frac{D_{1}}{1-x}\right) D_{1}^{\epsilon-1}\right]\right\}
\end{gathered}
$$

where $D_{1} \equiv x(1-x)$. The one-dimensional integral in the previous expression can be easily calculated and gives

$$
\begin{gathered}
\tilde{I}_{1}=\frac{\Gamma^{2}(\epsilon)}{\Gamma(2 \epsilon)}\left[(\psi(1-\epsilon)-\psi(1)-\psi(\epsilon)+\psi(2 \epsilon))\left(\psi(1-\epsilon)-\psi(1)-2(\psi(\epsilon)-\psi(2 \epsilon))+\ln \vec{k}_{2}^{2}\right)\right. \\
\left.+\psi^{\prime}(\epsilon)-2 \psi^{\prime}(2 \epsilon)+\psi^{\prime}(1-\epsilon)\right] .
\end{gathered}
$$

For the two-dimensional integral in (A.15),

$$
\begin{gathered}
\tilde{I}_{2}=\int_{0}^{1} d x \int_{0}^{1} \frac{d y}{1-y}\left[(\psi(1-\epsilon)-\psi(1))\left(y^{\epsilon} D_{y}^{\epsilon-1}-D_{1}^{\epsilon-1}\right)\right. \\
\left.\left.-\ln \left(\frac{D_{y}}{1-x}\right) y^{\epsilon} D_{y}^{\epsilon-1}+\ln \left(\frac{D_{1}}{1-x}\right) D_{1}^{\epsilon-1}\right]\right\},
\end{gathered}
$$

it is convenient to separate the integration region over $x$ into three parts, (i) $0<x<\delta$, (ii) $\delta<x<1-\delta$, (iii) $1-\delta<x<1$, and to calculate the three corresponding integrals in the limit $\delta \rightarrow 0$. In the integration region (i) it is possible to make the approximations $D_{y} \simeq(1-y) \vec{k}_{2}^{2}+x$ and $D_{1} \simeq x$ and a straightforward calculation leads to the following result, with accuracy up to $O\left(\epsilon^{0}\right)$ :

$$
\tilde{I}_{2}^{(i)} \simeq-\frac{2}{\epsilon^{3}}-\frac{1}{3} \ln ^{3} \delta-\psi^{\prime}(1)\left(\ln \delta-\frac{1}{\epsilon}\right)+\ln \vec{k}_{2}^{2}\left(-\frac{1}{\epsilon^{2}}+\frac{1}{2} \ln ^{2} \delta+\psi^{\prime}(1)\right) .
$$


Similarly, in the integration region (iii) it is possible to make the approximations $D_{y} \simeq$ $(1-y) \vec{k}_{2}^{2}+1-x$ and $D_{1} \simeq 1-x$ and to obtain, with accuracy up to $O\left(\epsilon^{0}\right)$ :

$$
\tilde{I}_{2}^{(i i i)} \simeq \psi^{\prime}(1) \ln \left(\frac{\vec{k}_{1}^{2}}{\delta}\right)+\frac{1}{2} \psi^{\prime \prime}(1) .
$$

Finally, in the integration region (ii) $\epsilon$ can be put equal to zero, since there are no divergences, and we obtain

$$
\begin{gathered}
\tilde{I}_{2}^{(i i)} \simeq \ln \delta\left[\frac{1}{2} \ln ^{2}\left(\frac{\vec{k}_{1}^{2}}{\delta}\right)+\frac{1}{2} \ln ^{2}\left(\frac{\vec{k}_{2}^{2}}{\delta}\right)+2 \psi^{\prime}(1)\right]-\frac{1}{2} \int_{\delta}^{1-\delta} \frac{d x}{D_{1}}\left[\ln ^{2} x-\ln ^{2}\left(\frac{D_{0}}{1-x}\right)\right] \\
+\int_{\delta}^{1-\delta} d x \ln \left(\frac{x}{1-x}\right) \ln \left(\frac{D_{1}}{D_{0}}\right)\left[\frac{1-2 x}{D_{1}}+\frac{\vec{k}_{1}^{2}-\vec{k}_{2}^{2}-(1-2 x)}{D_{1}-D_{0}}\right] \\
=\frac{1}{3} \ln ^{3} \delta-\frac{1}{2} \ln ^{2} \delta \ln \vec{k}_{2}^{2}+2 \psi^{\prime}(1) \ln \delta+2 \psi^{\prime \prime}(1) \\
+\frac{1}{2} \int_{0}^{1} \frac{d x}{x} \ln ^{2}\left(\frac{D_{0}}{\vec{k}_{2}^{2}}\right)+\frac{1}{2} \int_{0}^{1} \frac{d x}{1-x} \ln ^{2}\left(\frac{D_{0}}{\vec{k}_{1}^{2}}\right)+\ln \vec{k}_{2}^{2} \int_{0}^{1} \frac{d x}{x} \ln \left(\frac{D_{0}}{\vec{k}_{2}^{2}}\right)+\ln \vec{k}_{1}^{2} \int_{0}^{1} \frac{d x}{1-x} \ln \left(\frac{D_{0}}{\vec{k}_{1}^{2}}\right) \\
+\int_{0}^{1} \frac{d x}{1-x} \ln x \ln D_{0}-\int_{0}^{1} \frac{d x}{x} \ln x \ln \left(\frac{D_{0}}{\vec{k}_{2}^{2}}\right)-2 \int_{0}^{1} \frac{d x}{1-x} \ln (1-x) \ln \left(\frac{D_{0}}{\vec{k}_{1}^{2}}\right) \\
-\int_{0}^{1} d x \ln \left(\frac{x}{1-x}\right) \ln \left(\frac{D_{1}}{D_{0}}\right) \frac{\vec{k}_{1}^{2}-\vec{k}_{2}^{2}-(1-2 x)}{D_{0}-D_{1}},
\end{gathered}
$$

where $D_{0} \equiv x \vec{k}_{1}^{2}+(1-x) \vec{k}_{2}^{2}$ and we have used

$$
\int_{0}^{1} \frac{d x}{x} \ln (1-x)=-\operatorname{Li}_{2}(1)=-\psi^{\prime}(1), \quad \int_{0}^{1} \frac{d x}{x} \ln ^{2}(1-x)=2 \operatorname{Li}_{3}(1)=-\psi^{\prime \prime}(1) .
$$

Summing up the results in (A.16) and A.18)-(A.20) to obtain $\tilde{I}\left(\vec{k}_{1}^{2}, \vec{k}_{2}^{2}\right)$ with $O\left(\epsilon^{0}\right)$ accuracy, it is easy to check that all $\delta$-dependent terms disappear and that divergences cancel each other. The final result is the following:

$$
I\left(\vec{p}_{1}^{2}, \vec{p}_{2}^{2} ;\left(\vec{p}_{1}-\vec{p}_{2}\right)^{2}\right)=\tilde{I}\left(\vec{k}_{1}^{2}, \vec{k}_{2}^{2}\right)=\psi^{\prime}(1) \ln \vec{k}_{1}^{2}+[\text { one-dimensional integrals in }(\mathrm{A} .20)],
$$

with $\vec{k}_{1}$ and $\vec{k}_{2}$ defined as in (A.11) and $D_{1} \equiv x(1-x)$ and $D_{0} \equiv x \vec{k}_{1}^{2}+(1-x) \vec{k}_{2}^{2}$.

The third integral we should consider in this Appendix is $J\left(\vec{p}_{1}^{2}, \vec{p}_{2}^{2} ;\left(\vec{p}_{1}-\vec{p}_{2}\right)^{2}\right)$ defined in (2.26). Its calculation can be carried on following the same strategy as for $I\left(\vec{p}_{1}^{2}, \vec{p}_{2}^{2} ;\left(\vec{p}_{1}-\right.\right.$ $\left.\vec{p}_{2}\right)^{2}$ ). We will not present here the calculation, but merely show the final result, which reads

$$
\begin{gathered}
J\left(\vec{p}_{1}^{2}, \vec{p}_{2}^{2} ;\left(\vec{p}_{1}-\vec{p}_{2}\right)^{2}\right)=\frac{\left(\vec{p}_{1}-\vec{p}_{2}\right)^{2}}{\pi} \int d \vec{p} \frac{\ln \left(\vec{p}_{1}^{2} / \vec{p}^{2}\right) \ln \left(\vec{p}_{2}^{2} / \vec{p}^{2}\right)}{\left(\vec{p}-\vec{p}_{1}\right)^{2}\left(\vec{p}-\vec{p}_{2}\right)^{2}} \\
=\frac{1}{\pi} \int d \vec{k} \frac{\ln \left(\vec{k}_{1}^{2} / \vec{k}^{2}\right) \ln \left(\vec{k}_{2}^{2} / \vec{k}^{2}\right)}{\left(\vec{k}-\vec{k}_{1}\right)^{2}\left(\vec{k}-\vec{k}_{2}\right)^{2}}=\ln \left(\frac{\vec{k}_{1}^{2}}{\vec{k}_{2}^{2}}\right)\left[\int_{0}^{1} \frac{d x}{1-x} \ln \left(\frac{D_{0}}{\vec{k}_{1}^{2}}\right)-\int_{0}^{1} \frac{d x}{x} \ln \left(\frac{D_{0}}{\vec{k}_{2}^{2}}\right)\right]
\end{gathered}
$$




$$
\begin{gathered}
+\int_{0}^{1} \frac{d x}{x} \ln ^{2}\left(\frac{D_{0}}{\vec{k}_{2}^{2}}\right)+\int_{0}^{1} \frac{d x}{1-x} \ln ^{2}\left(\frac{D_{0}}{\vec{k}_{1}^{2}}\right) \\
-2 \int_{0}^{1} \frac{d x}{D_{1}-D_{0}} \ln \left(\frac{x}{1-x}\right) \ln \left(\frac{D_{1}}{D_{0}}\right)\left[(1-2 x)-\frac{D_{1}\left(\vec{k}_{1}^{2}-\vec{k}_{2}^{2}\right)}{D_{0}}\right],
\end{gathered}
$$

with the same notations as the previous calculation, i.e. with $\vec{k}_{1}$ and $\vec{k}_{2}$ defined as in (A.11) and $D_{1} \equiv x(1-x)$ and $D_{0} \equiv x \vec{k}_{1}^{2}+(1-x) \vec{k}_{2}^{2}$.

\section{B Appendix}

It is interesting to note (and can be useful for applications) that the integral $I$ entering the contribution $\mathcal{K}_{3}$ to the kernel is a totally symmetric function of the variables $\vec{q}_{1}^{2}, \vec{q}_{2}^{2}$ and $\vec{k}^{2}$. It is obvious from the representation

$$
I=\int_{0}^{1} \int_{0}^{1} \int_{0}^{1} \frac{d x_{1} d x_{2} d x_{3} \delta\left(1-x_{1}-x_{2}-x_{3}\right)}{\left(\vec{q}_{1}^{2} x_{1}+\vec{q}_{2}^{2} x_{2}+\vec{k}^{2} x_{3}\right)\left(x_{1} x_{2}+x_{1} x_{3}+x_{2} x_{3}\right)},
$$

which also can be useful in applications. One can easily check that this representation reproduces the original form, performing the integration over $x_{3}$, then making the change of variables

$$
\begin{gathered}
x=\frac{x_{2}}{x_{1}+x_{2}}, \quad z=\frac{x_{1} x_{2}}{x_{3}\left(1-x_{3}\right)+x_{1} x_{2}}=\frac{x_{1} x_{2}}{x_{1}\left(1-x_{1}\right)+x_{2}\left(1-x_{2}\right)-x_{1} x_{2}} \\
x_{1}=\frac{(1-x) z}{z+x(1-x)(1-z)}, \quad x_{2}=\frac{x z}{z+x(1-x)(1-z)}, \quad x_{3}=\frac{x(1-x)(1-z)}{z+x(1-x)(1-z)},
\end{gathered}
$$

with the Jacobian

$$
J\left(\frac{x_{1}, x_{2}}{x, z}\right)=\frac{z x(1-x)}{(z+x(1-x)(1-z))^{3}},
$$

and integrating over $z$.

Another useful representation is

$$
I=\int_{0}^{1} d x \int_{1}^{\infty} \frac{d t}{t} \frac{1}{\vec{q}_{1}^{2} x(1-x)(t-1)+\vec{q}_{2}^{2}(1-x)+\vec{k}^{2} x}
$$

Using this representation it is possible in a relatively simple way to express the contribution with $\mathcal{K}_{3}$ in the bootstrap condition in terms of a one-dimensional integral. Let us divide this contribution into several pieces:

$$
A \equiv \frac{1}{\pi} \int \frac{d^{2} q_{2}}{{\overrightarrow{q_{2}}}_{2}^{2} \vec{q}_{2}^{\prime 2}} \mathcal{K}_{3}=\left(A_{1}+A_{2}+A_{3}\right)+\left(\vec{q}_{1} \leftrightarrow \vec{q}_{1}^{\prime}\right)
$$

where

$$
A_{1}=\frac{1}{2 \pi} \int \frac{d^{2} q_{2}}{\vec{q}_{2}^{2} \vec{q}_{2}^{\prime 2}}\left[\vec{q}^{2}\left(\vec{k}^{2}-\vec{q}_{1}^{2}\right)+\vec{q}_{2}^{2}\left(\vec{q}_{1}^{2}-\vec{q}_{1}^{\prime 2}\right)\right] I
$$




$$
\begin{gathered}
A_{2}=\frac{1}{2 \pi} \int \frac{d^{2} q_{2}}{\vec{k}^{2} \vec{q}_{2}^{\prime 2}}\left[\vec{q}_{1}^{\prime 2}\left(\vec{q}_{2}^{2}-\vec{q}_{1}^{2}\right)+\vec{k}^{2}\left(\vec{q}_{1}^{2}-\vec{q}^{2}\right)\right] I \\
A_{3}=\frac{1}{2 \pi} \int \frac{d^{2} q_{2}}{\vec{q}_{2}^{2} \vec{k}^{2}} \vec{q}_{1}^{2}\left(\vec{q}_{1}^{2}-\vec{k}^{2}-\vec{q}_{2}^{2}\right) I .
\end{gathered}
$$

It is easy to see, with account of the relations $\vec{k}=\vec{q}_{1}-\vec{q}_{2}$ and $\vec{q}_{1,2}^{\prime}=\vec{q}-\vec{q}_{1,2}$, that

$$
A_{2}=A_{1}\left(\vec{q}_{1}^{\prime} \leftrightarrow-\vec{q}\right), \quad A_{3}=-A_{1}\left(\vec{q}_{1}^{\prime}=0\right)
$$

so that we need to calculate only $A_{1}$.

Let us write the Feynman parametrization in the form:

$$
\begin{gathered}
\frac{1}{\vec{q}_{2}^{2} \vec{q}_{2}^{\prime 2}\left(\vec{q}_{1}^{2} x(1-x)(t-1)+\vec{q}_{2}^{2}(1-x)+\vec{k}^{2} x\right)}=2 \int_{0}^{x} \frac{d z}{x} \int_{0}^{1} d v(1-v) \\
\times \frac{1}{\left(\vec{q}_{2}^{2}(1-v)(1-z / x)+\vec{q}_{2}^{\prime 2} v+\left(\vec{q}_{1}^{2} x(1-x)(t-1)+\vec{q}_{2}^{2}(1-x)+\vec{k}^{2} x\right)(1-v) z / x\right)^{3}} .
\end{gathered}
$$

Then, after the integration over $\vec{q}_{2}$ (of course, with account of the relation $\vec{k}=\vec{q}_{1}-\vec{q}_{2}$ ), the subsequent integrations over $v$ and $t$ are quite simple and we arrive at

$$
\begin{gathered}
A_{1}=\frac{1}{2} \int_{0}^{1} \frac{d x}{x} \int_{0}^{x} \frac{d z}{\left(\vec{q}^{2}-\vec{q}_{1}^{2} z\right)(1-z)+\vec{q}_{1}^{2} z}\left[\left(\left(\vec{q}^{2}+\vec{q}_{1}^{2}-\vec{q}_{1}^{\prime 2}\right) x-2 \vec{q}^{2}\right)\right. \\
\times\left(\frac{1}{x-z} \ln \left(\frac{1-z}{1-x}\right)-\frac{\vec{q}_{1}^{2} z}{\vec{q}^{2}(1-z)-\vec{q}_{1}^{2} z(1-x)+\vec{q}_{1}^{\prime 2} z} \ln \left(\frac{\vec{q}^{2}(1-z)+\vec{q}_{1}^{\prime 2} z}{\vec{q}_{1}^{2} z(1-x)}\right)\right) \\
\left.+\left(\vec{q}^{2}+\vec{q}_{1}^{2}-\vec{q}_{1}^{\prime 2}\right) \ln \left(\frac{\vec{q}^{2}(1-z)+\vec{q}_{1}^{\prime 2} z}{\vec{q}_{1}^{2} z(1-z)}\right)\right] .
\end{gathered}
$$

The result of the further integration of the total integrand in any of the variables $x, z$ can not be expressed in terms of elementary functions. But we can perform the integration in such a way: integrate over $x$ all terms except those which contain $\ln (1-x)$; these terms must be integrated over $z$ first. The terms with the denominator $x-z$ must be regularized (for example, we can perform the integration over the region $z \geq 0, x \leq 1, x-z \geq \delta \rightarrow 0$. The result is

$$
\begin{gathered}
A_{1}=\frac{1}{2} \int_{0}^{1} \frac{d z}{\vec{q}^{2}(1-z)+\vec{q}_{1}^{\prime 2} z-\vec{q}_{1}^{2} z(1-z)}\left[\left(\frac{2 \vec{q}^{2} \vec{q}_{1}^{2} z}{\vec{q}^{2}(1-z)+\vec{q}_{1}^{\prime 2} z-\vec{q}_{1}^{2} z}\right.\right. \\
\left.+\vec{q}^{2}+\vec{q}_{1}^{2}-\vec{q}_{1}^{\prime 2}\right) \ln \left(\frac{\vec{q}^{2}(1-z)+\vec{q}_{1}^{\prime 2} z-\vec{q}_{1}^{2} z(1-z)}{z\left(\vec{q}^{2}(1-z)+\vec{q}_{1}^{\prime 2} z\right)}\right) \ln \left(\frac{\vec{q}^{2}(1-z)+\vec{q}_{1}^{\prime 2} z}{\vec{q}_{1}^{2} z}\right) \\
\left.+\left(\frac{2 \vec{q}^{2}}{z}-\vec{q}^{2}-\vec{q}_{1}^{2}+\vec{q}_{1}^{\prime 2}\right) \ln (1-z) \ln \left(\frac{\vec{q}^{2}(1-z)+\vec{q}_{1}^{\prime 2} z-\vec{q}_{1}^{2} z(1-z)}{\vec{q}^{2}(1-z)}\right)\right] .
\end{gathered}
$$

It is rather easy to obtain from this representation exact value of $A_{3}$. Putting $\vec{q}^{2}=$ $\vec{q}_{1}^{2}, \quad \vec{q}_{1}^{\prime 2}=0$ we have

$$
A_{3}=-2 \zeta(3) .
$$


It is not difficult as well to calculate the asymptotic behavior of $A_{1}$ and $A_{2}$ at $\vec{q}^{2} \gg \vec{q}_{1}^{2}, \quad \vec{q}^{2} \gg$ $\vec{q}_{1}^{\prime 2}$. We obtain

$$
\begin{gathered}
A_{1} \simeq-\frac{\zeta(2)}{2} \ln \left(\frac{\vec{q}_{1}^{2}}{\vec{q}_{1}^{\prime 2}}\right), \\
A_{2} \simeq-\frac{1}{4} \ln ^{2}\left(\frac{\vec{q}^{2}}{\vec{q}_{1}^{\prime 2}}\right) \ln \left(\frac{\vec{q}^{2}}{\vec{q}_{1}^{2}}\right)-\frac{\zeta(2)}{2}\left(\ln \left(\frac{\vec{q}^{2}}{\vec{q}_{1}^{2}}\right)+2 \ln \left(\frac{\vec{q}^{2}}{\vec{q}_{1}^{\prime 2}}\right)\right)-\zeta(3),
\end{gathered}
$$

so that

$$
A \simeq-\frac{1}{4} \ln \left(\frac{\vec{q}^{2}}{\vec{q}_{1}^{\prime 2}}\right) \ln \left(\frac{\vec{q}^{2}}{\vec{q}_{1}^{2}}\right)\left(\ln \left(\frac{\vec{q}^{2}}{\vec{q}_{1}^{2}}\right)+\ln \left(\frac{\vec{q}^{2}}{\vec{q}_{1}^{\prime 2}}\right)\right)-\frac{3 \zeta(2)}{2}\left(\ln \left(\frac{\vec{q}^{2}}{\vec{q}_{1}^{2}}\right)+\ln \left(\frac{\vec{q}^{2}}{\vec{q}_{1}^{\prime 2}}\right)\right)-6 \zeta(3) .
$$

For calculation of the discontinuity of $A$ at $q^{2}=-\vec{q}^{2} \geq 0$ the last representation of $A_{3}$ is not very convenient, since its analytical properties are not simple. In fact, it is more convenient to do one step back and to use the representation in the form of the two-dimensional integral.

\section{Appendix}

Another way of calculation of the discontinuity of $A$ at $q^{2}=-\vec{q}^{2} \geq 0$ is to rewrite the integral over $q_{2}$ in Minkowski space and to use the Cutkosky rules for the calculation of the discontinuity. Let us use the representation

$$
I=\int_{0}^{1} d x \int_{0}^{\infty} d z \frac{1}{z-k^{2} x(1-x)-i 0} \frac{1}{z-q_{1}^{2}(1-x)-q_{2}^{2} x-i 0},
$$

where $k, q_{1}, q_{2}$ are considered as vectors in the two-dimensional Minkowski space, i.e. $k^{2}=-\vec{k}^{2}, q_{1}^{2}=-\vec{q}_{1}^{2}, q_{2}^{2}=-\vec{q}_{2}^{2}$. This representation can be used for arbitrary values of $k^{2}, q_{1}^{2}, q_{2}^{2}$. Analogous representation can be written for $I\left(q_{i} \leftrightarrow q_{i}^{\prime}\right)$. It permits to rewrite the integral with $\mathcal{K}_{3}$ in the bootstrap relation in the form

$$
A=\frac{1}{i \pi} \int \frac{d^{2} q_{2}}{\left(q_{2}^{2}+i 0\right)\left(\left(q-q_{2}\right)^{2}+i 0\right)} \mathcal{K}_{3}
$$

where now

$$
d^{2} q_{2}=d q_{2}^{(0)} d q_{2}^{(1)}, \quad q_{2}^{2}=\left(q_{2}^{(0)}\right)^{2}-\left(q_{2}^{(1)}\right)^{2},
$$

etc., which determines $A$ as the function of $q_{1}^{2}, q_{1}^{\prime 2}$ and $q^{2}$ for arbitrary values of these variables. For $q_{1}^{2} \equiv-\vec{q}_{1}^{2} \leq 0, q_{1}^{\prime 2} \equiv-\vec{q}_{1}^{\prime 2} \leq 0$ and $q^{2} \equiv-\vec{q}^{2} \leq 0$ it is just the function entering (2.24), that is easily seen by making the Wick rotation of the contour of integration over $q_{2}^{(0)}$. We are interested in the region $q_{1}^{2} \leq 0, q_{1}^{\prime 2} \leq 0$ and $q^{2} \geq 0$. According to the Cutkosky rules, the discontinuity of $A$ related to the terms with $I$ is determined by the two cuts, with the contributions obtained by the substitutions:

$$
\frac{1}{\left(q_{2}^{2}+i 0\right)\left(\left(q-q_{2}\right)^{2}+i 0\right)} \rightarrow(-2 \pi i)^{2} \delta\left(q_{2}^{2}\right) \delta\left(\left(q-q_{2}\right)^{2}\right)
$$


and

$$
\frac{1}{\left(z-q_{1}^{2}(1-x)-q_{2}^{2} x-i 0\right)\left(\left(q-q_{2}\right)^{2}+i 0\right)} \rightarrow-(-2 \pi i)^{2} \delta\left(z-q_{1}^{2}(1-x)-q_{2}^{2} x\right) \delta\left(\left(q-q_{2}\right)^{2}\right) .
$$

Using these rules and removing the $\delta$-functions by the integration over $q_{2}$ (the most appropriate system for this is $\left.q^{(1)}=0, q^{2}=\left(q^{(0)}\right)^{2}\right)$, we obtain

$$
\frac{\Im A}{\pi}=f_{1}+f_{2}+\left(q_{1}^{2} \leftrightarrow q_{1}^{\prime 2}\right)
$$

where the contributions $f_{1}$ and $f_{2}$ come from the first and second cuts, respectively. We obtain for them

$$
f_{1}=\frac{1}{2} \int_{0}^{1} d x \int_{0}^{\infty} d z \frac{\vec{q}_{1}^{2}-\kappa^{+}}{\left(z+\kappa^{+} x(1-x)\right)\left(z+\vec{q}_{1}^{2}(1-x)\right)}+\left(\kappa^{+} \rightarrow \kappa^{-}\right),
$$

where $\kappa^{ \pm}$are the values of $-k^{2}$ on the mass shell $q_{2}^{2}=0,\left(q-q_{2}\right)^{2}=0$, related to the two possible solutions for $q_{2}^{(1)}$ :

$$
\kappa^{ \pm}=\frac{1}{2}\left(q^{2}+\vec{q}_{1}^{2}+\vec{q}_{1}^{\prime 2} \pm \sqrt{\left(q^{2}+\vec{q}_{1}^{2}+\vec{q}_{1}^{\prime 2}\right)^{2}-4 \vec{q}_{1}^{2} \vec{q}_{1}^{\prime 2}}\right)
$$

and

$$
\begin{gathered}
f_{2}=\frac{1}{2} \int_{0}^{1} d x \int_{0}^{\infty} \frac{d z \theta\left(q^{2} x-\vec{q}_{1}^{2}(1-x)-z\right)}{z+\tilde{\kappa}^{+} x(1-x)}\left[\frac{\kappa^{+}-\vec{q}_{1}^{2}}{z+\vec{q}_{1}^{2}(1-x)}\right. \\
\left.+\frac{\kappa^{+}\left(q^{2}+\vec{q}_{1}^{2}\right)-\vec{q}_{1}^{2} \vec{q}_{1}^{2}}{x q^{2} \tilde{\kappa}^{+}}\right]+\left(\kappa^{+} \rightarrow \kappa^{-}\right),
\end{gathered}
$$

where $\tilde{\kappa}^{ \pm}$are given by values of $-k^{2}$ on the mass shell $z-q_{1}^{2}(1-x)-q_{2}^{2} x=0,\left(q-q_{2}\right)^{2}=0$, so that

$$
\tilde{\kappa}^{ \pm}=\kappa^{ \pm}\left(1-\frac{z+\vec{q}_{1}^{2}(1-x)}{q^{2} x}\right)+\vec{q}_{1}^{\prime 2} \frac{z+\vec{q}_{1}^{2}(1-x)}{q^{2} x} .
$$

The integration over $z$ is quite elementary. Making subsequent integration over $x$ one has to pay attention that the contributions $f_{1,2}$ are separately divergent at $x=1$. The cancellation of the divergent terms is done easily and unambiguously. After that, a straightforward integration gives

$$
\begin{gathered}
\frac{\Im A}{\pi}=\operatorname{Li}_{2}\left(\frac{\kappa^{+}-\vec{q}_{1}^{2}}{q^{2}+\vec{q}_{1}^{2}}\right)+\operatorname{Li}_{2}\left(\frac{\kappa^{-}-\vec{q}_{1}^{2}}{q^{2}+\vec{q}_{1}^{2}}\right)+\operatorname{Li}_{2}\left(\frac{\vec{q}_{1}^{2}}{q^{2}+\vec{q}_{1}^{2}}\right)-\zeta(2)+\frac{1}{8} \ln ^{2}\left(\frac{\kappa^{+}}{q^{2}}\right) \\
+\frac{1}{8} \ln ^{2}\left(\frac{\kappa^{-}}{q^{2}}\right)+\frac{1}{2} \ln \left(\frac{q^{2}+\vec{q}_{1}^{2}}{q^{2}}\right) \ln \left(\frac{q^{2}\left(q^{2}+\vec{q}_{1}^{2}\right)^{3}}{\left(\vec{q}_{1}^{2} \vec{q}_{1}^{\prime 2}\right)^{2}}\right)+\frac{1}{4} \ln \left(\frac{\vec{q}_{1}^{2}}{q^{2}}\right) \ln \left(\frac{\vec{q}_{1}^{\prime 2}}{q^{2}}\right)+\left(q_{1}^{2} \leftrightarrow q_{1}^{\prime 2}\right) .
\end{gathered}
$$

This expression can be considerably simplified using the identity

$$
\operatorname{Li}_{2}\left(\frac{\kappa^{+}-\vec{q}_{1}^{2}}{q^{2}+\vec{q}_{1}^{2}}\right)+\operatorname{Li}_{2}\left(\frac{\kappa^{-}-\vec{q}_{1}^{2}}{q^{2}+\vec{q}_{1}^{2}}\right)+\frac{1}{2} \ln \left(\frac{\kappa^{-}}{q^{2}}\right) \ln \left(\frac{\kappa^{+}}{q^{2}}\right)+\left(q_{1}^{2} \leftrightarrow q_{1}^{\prime 2}\right)
$$




$$
=\zeta(2)-\operatorname{Li}_{2}\left(\frac{\vec{q}_{1}^{2}}{q^{2}+\vec{q}_{1}^{2}}\right)-\frac{1}{2} \ln \left(\frac{q^{2}+\vec{q}_{1}^{2}}{q^{2}}\right) \ln \left(\frac{q^{2}\left(q^{2}+\vec{q}_{1}^{2}\right)^{3}}{\left(\vec{q}_{1}^{2} \vec{q}_{1}^{\prime 2}\right)^{2}}\right)+\left(q_{1}^{2} \leftrightarrow q_{1}^{\prime 2}\right),
$$

which is not evident, of course, but can be proved, for example, considering both sides of the identity as the functions of $\vec{q}_{1}^{2}$ (evidently, taking into account the dependence of $\kappa^{ \pm}$from $\vec{q}_{1}^{2}$ )

and comparing their derivatives and their limits at $\vec{q}_{1}^{2} \rightarrow 0$. Using this identity we obtain finally

$$
\frac{\Im A}{\pi}=-\frac{3}{2} \ln \left(\frac{\kappa^{-}}{q^{2}}\right) \ln \left(\frac{\kappa^{+}}{q^{2}}\right)+\frac{1}{4} \ln ^{2}\left(\frac{\vec{q}_{1}^{2} \vec{q}_{1}^{\prime 2}}{\left(q^{2}\right)^{2}}\right)+\frac{1}{2} \ln \left(\frac{\vec{q}_{1}^{2}}{q^{2}}\right) \ln \left(\frac{\vec{q}_{1}^{\prime 2}}{q^{2}}\right) .
$$

Of course, the same result follows from the representation of $A$ obtained in Appendix B.

\section{References}

[1] V.S. Fadin, E.A. Kuraev and L.N. Lipatov, Phys. Lett. B60 (1975) 50; E.A. Kuraev, L.N. Lipatov and V.S. Fadin, Zh. Eksp. Teor. Fiz. 71 (1976) 840 [Sov. Phys. JETP 44 (1976) 443]; 72 (1977) 377 [45 (1977) 199]; Ya.Ya. Balitskii and L.N. Lipatov, Sov. J. Nucl. Phys. 28 (1978) 822.

[2] V.S. Fadin and L.N. Lipatov, Phys. Lett. B429 (1998) 127.

[3] M. Ciafaloni and G. Camici, Phys. Lett. B430 (1998) 349.

[4] V.S. Fadin and R. Fiore, Phys. Lett. B440 (1998) 359.

[5] V.S. Fadin, R. Fiore and A. Papa, Phys. Rev. D60 (1999) 074025.

[6] V.S. Fadin and D.A. Gorbachev, Pis'ma v Zh. Eksp. Teor. Fiz. 71 (2000) 322 [JETP Letters 71 (2000) 222]; Phys. Atom. Nucl. 63 (2000) 2157 [Yad. Fiz. 63 (2000) 2253].

[7] V.S. Fadin, R. Fiore, M.I. Kotsky and A. Papa, Phys. Rev. D61 (2000) 094005.

[8] V.S. Fadin, R. Fiore, M.I. Kotsky and A. Papa, Phys. Rev. D61 (2000) 094006.

[9] V.S. Fadin and A.D. Martin, Phys. Rev. D60 (1999) 114008.

[10] V. Fadin, D. Ivanov and M. Kotsky, In: New Trends in High-Energy Physics, Ed. L.L. Jenkovszky, Kiev, 2000, pp. 190-194, hep-ph/0007119.

[11] J. Bartels, S. Gieseke and C.F. Qiao, Phys. Rev. D63 (2001) 056014, hep-ph/0009102.

[12] V. Fadin, D. Ivanov, M. Kotsky, hep-ph/0106099.

[13] J. Bartels, S. Gieseke, A. Kyrieleis, Phys. Rev. D65 (2000) 014006, hep-ph/0107152.

[14] Ya.Ya. Balitskii, L.N. Lipatov and V.S. Fadin, in Proceedings of Leningrad Winter School on Physics of Elementary Particles, Leningrad, 1979, 109 (in Russian).

[15] V.S. Fadin, R. Fiore and M.I. Kotsky, Phys. Lett. B494 (2000) 100, hep-ph/0007312. 
[16] V.S. Fadin, R. Fiore and A. Papa, Phys. Rev. D63 (2001) 034001, hep-ph/0008006.

[17] to be published.

[18] M. Braun, hep-ph/9901447.

[19] M. Braun and G.P. Vacca, Phys. Lett. B447 (2000) 156.

[20] V.S. Fadin, R. Fiore, M.I. Kotsky and A. Papa, Phys. Lett. B495 (2000) 329, hep-ph/0008057.

[21] M. Braun and G.P. Vacca, Phys. Lett. B454 (1999) 319.

[22] V.S. Fadin, Zh. Eksp. Teor. Fiz. Pis'ma 61 (1995) 342; V.S. Fadin, R. Fiore and A. Quartarolo, Phys. Rev. D53 (1996) 2729; M.I. Kotsky and V.S. Fadin, Yad. Fiz. 59 (6) (1996) 1; V.S. Fadin, R. Fiore and M.I. Kotsky, Phys. Lett. B359 (1995) 181.

[23] V.S. Fadin, R. Fiore and M.I. Kotsky, Phys. Lett. B387 (1996) 593, hep-ph/9605357.

[24] J. Blumlein, V. Ravindran and W.L. van Neerven, Phys. Rev. D58 (1998) 091502, hep-ph/9806357.

[25] V. Del Duca and E.W.N. Glover, JHEP 0110 (2001) 035, hep-ph/0109028. 